\title{
Ang Kasalimuotan ng Pagiging Fangirl: llang Alternatibong Pagtanaw sa Mundo ng Kababaihang Paghanga
}

Andrea Anne I. Trinidad

\begin{abstract}
Abstrakt
Malaki ang papel ng midya sa paghubog ng popular na kamalayan hinggil sa mga babaeng tagahanga. Sa artikulasyong nagmumula sa labas, hindi na bagong maisadlak sa representasyong patolohiko ang mga tagahanga na muling binalingan at sinandigan ng pelikulang humalaw ng pamagat sa identidad na madalas niyayakap ng mga tagahangang babae_-ang Fan Girl (Jadaone, 2020). Sa pamamagitan ng paglalatag ng pagtatalabang panlabas at panloob na sumisipat at nagbibigay-pagkakakilanlan sa identidad na ito, patitingkarin ang bisa ng fandom bilang sityong maaaring pag-ugatan ng alternatibong diskursong hindi lamang umaalagwa sa hegemonikong pagkilala sa mga fangirl, kundi nag-aalok din ng artikulasyon hinggil sa pansariling nosyon kung papaano maipapahayag ang kababaihang paghanga. Naka-angkla sa praktika ng fangirling, ilalahad ng papel ang mga mekanismong sinusuong ng fandom upang pasubalian ang talamak na representasyon, at ipakilala ang sariling identidad ng komunidad na patuloy na kasangkot sa paglikha ng mapagpalayang pagtanaw sa kasarian at seksuwalidad.
\end{abstract}

Keywords: Fangirl, Fan Girl (Jadaone, 2020), Praktika ng fangirling, Kababaihang Paghanga, Fandom 


\title{
Plaridel Open Access Policy Statement
}

As a service to authors, contributors, and the community, Plaridel: A Philippine Journal of Communication, Media, and Society provides open access to all its content. To ensure that all articles are accessible to readers and researchers, these are available for viewing and download (except Early View) from the Plaridel journal website, provided that the journal is properly cited as the original source and that the downloaded content is not modified or used for commercial purposes. Plaridel, published by the University of the Philippines College of Mass Communication is licensed under Creative Commons Attribution-NonCommercial-NoDerivatives 4.0 International License (https://creativecommons.org/ licenses/by-nc-nd/4.0/legalcode).

\section{How to cite this article in APA}

Trinidad, A. A. I. (2021). Ang kasalimuotan ng pagiging fangirl: Ilang alternatibong pagtanaw sa mundo ng kababaihang paghanga. Plaridel, 18(1), 147-180. https://doi.org/10.52518/2021.18.1-07trindd

\begin{abstract}
Mainstream media has played a vital role in shaping popular consciousness about female fans. Such articulations that emanate from outsiders oftentimes adhere to pathological representation that was revisited and compounded by a recent film that adapted for its title the identity embraced by female fans-being a Fan Girl (Jadaone, 2020). Through the analysis of conflicting views from outside and inside the community that interpret a fangirl's identity, the paper will highlight the fandom's efficacy as a site where alternative discourse arise that does not just oppose the hegemonic recognition against fangirls, but also offers an articulation how the community developed its notion of female fan expression. Anchored to the practice of fangirling, the paper will show mechanisms fans undergo to object the existing deep-rooted representation and exhibit the identity of a community that has long been involved in the creation of a more liberating view of gender and sexuality.
\end{abstract}


Tila matagal nang nakakintal sa pag-iisip ng madla na palaging babae ang isang tagahanga. ${ }^{1}$ Bukod sa pagtratong may ilang espesipikong anyo ng kulturang popular na alok partikular na para sa mga kababaihan na pinag-ugatan ng simulain ng pag-iral at pag-aaral ng mga babaeng tagahanga (Bacon-Smith, 1992; Jenkins, 1992; Penley, 1992), may palasak na ring paraan ng pagkilala sa kanila. Sa palaging pagluluklok ng katawan ng babaeng tagahanga sa mga biswal na anyo (Lar. 1), dagdag pa ang kanilang pagiging sentral na karakter sa mga mitong pinagpapasa-pasa tulad ng lokal na penomena ng mga Noranian (Trinidad, 2018, pp.1 47-148) o pandaigdigang penomena ng Beatlemania (Blake, 2020) bilang mga halimbawa, pamilyar nang naisasandig ang kanilang pisikalisasyon at representasyon sa katangiang "excess" (Scott, 2019, p. 231).

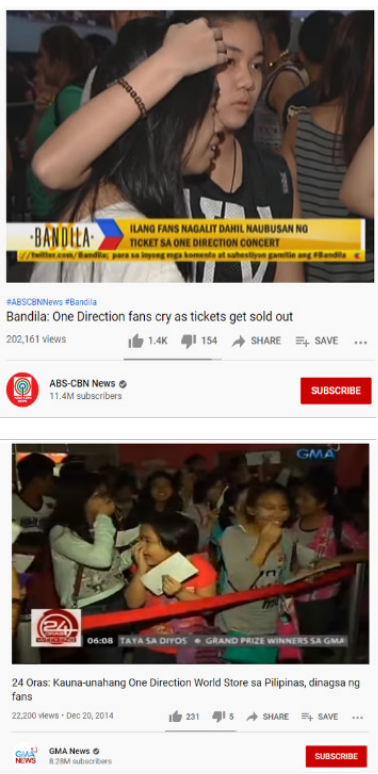

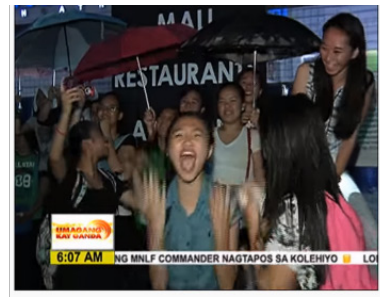

Local celebs enjoy One Direction concert
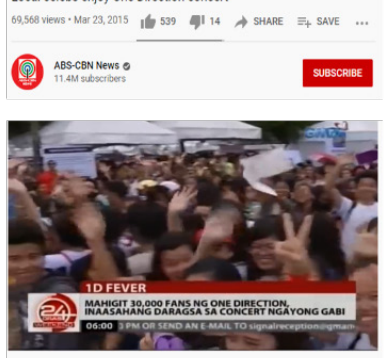

Mahigt 30,000 fans $n g$ One Direction, inaasahang daragsa sa concert ngayong gabi

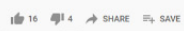

niture camanems o

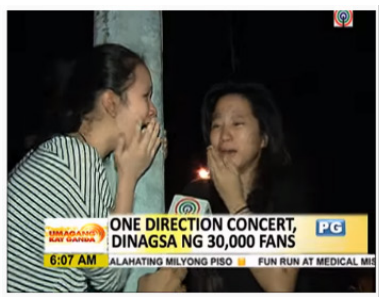

Local celebs enjoy One Direction concert
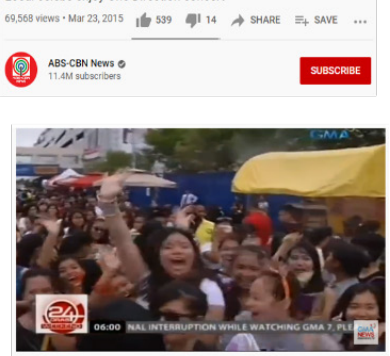

Mahigit 30,000 fans ng One Direction, inaasahang daragsa sa concer ngayong gabi

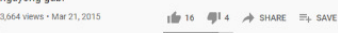

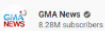

Larawan 1. Bagaman halaw ang mga retrato sa mga balitang inere sa telebisyon na sabay ring inilagak online (ABS-CBN News, 2014; ABS-CBN News, 2015; GMA News, 2014; GMA News, 2015), madaling maipagpalagay na sa ganitong paraan din madalas itampok ang mga babaeng tagahanga bilang mga histerikal na katawang dumudumog, tumitili, kumakawag, humahagulgol, nagwawala, at naglulupasay sa pelikula man, patalastas, at maging sa ilang dokumentaryong biyograpiko na nakasentro sa pamamayagpag ng karera ng hinahangaang idolo.

Dahil tinatayang palaging sumosobra, naikakawing ang ekspresyon ng kanilang paghanga sa mga aktong wala sa katwiran. Madaling dumudulas ang nakaririnding pagtili sa obsesyon, ang nag-uumapaw na admirasyon sa obheto ng paghanga bilang pagkahumaling dito, at ang labis na pagaalay ng atensiyon sa destruktibong pagtatali ng sarili sa iniidolo at 
agarang pagsasawalambahala ng sariling intelek at dangal. Bagaman agarang taglay ng pag-akap sa pagiging tagahanga ang pagpapagana ng ahensiyang nakatuon sa malayang pagpili sa idolong susuportahan, kaagad nasasadlak sa posisyong dehado ang isang babaeng tagahanga mula sa mga umiiral na estereotipong pagpapalagay. Isang pagsasaposisyong kagyat na napatitingkad ng terminong masaklaw nang ginagamit-ang taguring "fangirl," na hayagang may pagtuon sa kultural na kategorya ng kasarian at edad kasama ang matagal nang prehuwisyong dumidikta sa pagiging babae at pagiging bata.

Suson-suson sa gayon ang nalilikhang implikasyon ng makakasariang nosyon ng "fangirl" bilang taguri. Sa malawakang obserbasyon ni Yve Blake (2020) na nakasalig sa pag-iral ng dikotomiyang fangirl at fanboy, palaging dehado ang mga fangirl na agad nababakasán ng mga katangiang "crazy, psycho, scary, and a bit much" (4:37-4:42) sa tuwing titili sa harap ng idolo kung ikokompara sa mga sports fanboy na kakikitahan ng parehong pagkilos ngunit tatanawin bilang nagtataglay lamang ng normal na reaksiyon sa laro. Kung itutuloy ang paglalatag ng dikotomiya, nasasadlak din sa pagkadehado ang mga fangirl ng mga kultural na materyal na tradisyonal nang itinuturing bilang "panlalaki" tulad ng komiks at larong pampalakasan. Sapagkat matagal nang tinanggap ng lipunan na nabibilang lamang ang mga fangirl sa arena ng soap opera, romance, at boyband, naniniwala si Suzanne Scott (2019) na may nagaganap na "war on fangirls" at sa isang banda "war on women" din (p.20) sa industriya ng komiks na bukod sa pamamayagpag ng bansag na "fake geek girls" na tila kinukuwestiyon ang awtentisidad ng kababaihang paghanga, isinisisi din sa mga ito ang pagbaba ng bentahan sa industriya sa pagiging kritikal ng mga ito laban sa mga komiks na may hegemoniko pa ring representasyon ng kanilang kasarian (Scott, 2019, pp. 1-2). Upang maigiit ang sabay na pagiging babae at tagahanga, may ilang fangirl din ng football na pilit itinatago ang kanilang identidad sa pamamagitan ng anonymous na pagdulog sa mga online forum na sinasabayan din ng kanilang pakikigamit ng mga pahayag laban sa mga babae nang hindi mapaghalataang ipinagtatanggol ang ang kinabibilangang kasarian (Cook \& Hynes, 2014, p. 87).

Kung bubusisiin pang lalo ng makakasariang nosyon ng paghanga sa labas ng fangirl-fanboy bilang binary, makikitang napapailalim din ang ilang lalaking tagahanga sa hegemoniyang takda ng patriyarka na pilit iwinawaksi ang pagiging likas ng emosyon. Sa pag-aaral ni Mel Stanfill (2011) sa kultural na representasyon ng mga tagahanga sa parehong piksiyonal at di-piksiyonal na anyong pang-midya, nagkakasundo ang mga ito sa pagtrato sa mga fanboy bilang nagtataglay ng di-sapat na pagkiling sa "normative masculinity" (para. 3.5). Mula sa kanilang pisikalisasyon bilang sobra sa timbang na dulot 
ng di-pagiging aktibo, tungo sa mga natatanggap na batikos tulad ng mga insultong "ladies," "Spice Girls," at "pussies," hanggang sa pagdududang makabubuo sila ng heteronormatibo at romantikong relasyon (para. 3.2), hayag na nagagamit sa mundo ng paghanga ang kasarian at ang tradisyonal na pagtanaw dito upang magsama o magsantabi ng mga posisyon at suheto sa loob ng fandom (Scott, 2019, p. 6).

Kaya naman maski napatunayan ng pangkat-pangkat ng fangirl ang bisa ng kanilang paghanga nitong nakaraang taon-nariyan ang politikal at kolektibong pagkilos ng KathNiel fans nangilunsad nitoang\#WeBlockAsOne laban sa mga "troll" na pabor sa pagsasara ng ABC-CBN (\#WeBlockAsOne: KathNiel Fans Lead Campaign Vs. Trolls, 2020), maging ang transnasyonal na K-pop fandom na nakianib sa mga Tiktok users upang matiyak na hindi dadagsain ng mga tagasuporta ang kampanya ni Trump (Lorenz et al., 2020), nakapataw pa rin sa kanila ang mga patolohikong palatandaang nakatali sa pagiging femenisado (Scott, 2019, p. 16). Naniniwala si Stanfill (2011) na malaki ang ambag ng mga diskursong isinusulong ng mainstream media sa umiiral na konstuksiyon ng "fan." Dahil kagyat itong lumilikha ng representasyon at, sa isang banda, realidad kung paano maaaring tanawin ang mga tagahanga (para. 2.3), mainam gawing lunsaran ng higit na masalimuot na pagkilala sa mga kababaihang tagahanga ang Fan Girl (Jadaone, 2020) bilang pelikulang nagsara ng taong 2020 na siyang parehong taon kung kailan lumikha ng hiwalay na diskurso ang KathNiel fandom at Kpop fans sa nosyon ng paghanga.

Sa pag-uungkat sa paraan kung paano nililikha ng pelikulang Fan Girl (Jadaone, 2020) ang representasyon ng babaeng paghanga sa pagsubaybay nito sa pagkilos at pagpapasya ng karakter ni Jane (Charlie Dizon) bilang tampok na fangirl ni Paulo Avelino, isisiwalat ng papel ang naging pagkiling ng pelikula sa "patolohiko at hegemoniko" pa ring pagkilala sa isang babaeng tagahanga (Trinidad, 2021, para. 5). Malaon, itatahi sa pakikiisang isinagawa ng Fan Girl (Jadaone, 2020) sa panlabas na tradisyon ng femenisadong pagtanaw sa fan ang mga panloob na obserbasyong umuugat sa matalik na paglublob ng manunulat sa mundo ng paghanga na hindi lamang makapagaalok ng alternatibong diskursong tinataya na aalagwa sa dominanteng pagkilala, kundi makapagsisiwalat ng pag-unawang umuugat mismo sa masalimuot na artikulasyon ng mga fangirl hinggil sa kanilang niyayakap na identidad. Sa paglalatag ng mga nakagawiang praktis ng komu-komunidad na fangirl, partikular na yaong nakahanap ng puwang sa espasyong online tulad ng Twitter, kikilatisin ang mga tendensiyang sinusuong nila na makapagpapatingkad ng mga sityo ng negosasyon na kinasasandigan ng pagkawala ng mga fangirl sa umiiral na dominanteng paglalarawan sa kanila. 


\section{Fangirl, Fandom, at ang Dikta ng Espasyo}

Binubuksan ang pelikulang Fan Girl (Jadaone, 2020) sa mainam na pagsasaposiyon ng isang babaeng tagahanga. Bagaman malinaw para sa pelikula ang layunin nitong makapagtampok ng kakila-kilabot na kahihinatnan ng sinumang may labis na paghanga na akma sa kontekstong politikal ng bansa sa kasalukuyan, naging maingat ang pelikula sa panimulang pagsasakarakter kay Jane bilang isang marubdob na fangirl ng artistang si Paulo Avelino na ginampanan din ng naturang aktor. Suot-suot ang malinis na unipormeng nababagayan ng ayós na ayós na buhok, kagyat ipapamalay ng pelikula sa mga manonood ang katayuang fangirl ng dalaga na siyang magmamadaling makaposisyon sa loob ng jeep upang mapanood ang trailer ng If We Fall in Love na bagong pelikulang pinagbibidahan ng idolo. Sa panimulang eksenang ito, mamamalas din ng mga manonood ang ilang posibleng dahilan kung bakit fangirl si Jane ni Avelino na siyang ipinipinta bilang mabango, makisig, at maginoo-isang artistang hindi nalalayo sa deskripsiyon ni Joi Barrios (2015) sa mga gumaganap na leading men na maituturing bilang "makabagong Prince Charming" at "siyotang pangarap" (p. 280).

Interesante ang pagpipinta ng isang fangirl sa panimulang eksenang ito. Bukod sa tinatalikdan nito ang malimit na pagkilala sa tagahangang nahuhubaran ng personal na buhay upang ilublob sa representasyong nabubuhay lamang upang ipakita ang debosyon at labis na pagpapahalaga sa iniidolo (Jenkins, 1992, p. 10), ibinabalik sa pagsasakatawan ng isang fangirl ang responsabilidad nito bilang isang mag-aaral. Bagaman masasabing ang paglulugar kay Jane bilang isang mag-aaral sa hay-iskul ay nakapagpapatingkad lamang ng diskursong palaging bata ang isang tagahanga, at kung gayo'y mailalarawan bilang "emotionally and intellectually immature" (Jenkins, 1992, p. 10), nababalanse ang eksena sa pagpapamalas ng ahensiya ni Jane bilang isang fangirl-ahensiya na siyang magpapakilos sa kaniyang ilaan ang ilang saglit ng nakababagot na pagkokomyut sa pagpiling bumabad sa masayang mundo ng paghanga habang pilit na naiimpit ang kilig sapagkat may sapat na kamalayan sa dikta ng jeep bilang isang pampublikong espasyong may kaayusang ipinapatupad. Maski lubog sa lugod na handog ng sandaling napapanood ang idolo sa cellphone, napagiingatan pa rin ni Jane ang sariling dangal sa hindi pagsambulat ng kaniyang emosyon.

Sa likod ng katotohanang may sapat na panghahawak ang isang fangirl sa kaniyang emosyon na nahahagip ng pelikula, katotohanan ding may ligtas na espasyong kakanlong sa katanggap-tanggap na pagpapahayag nito na mababakás din sa unang sampung minuto ng Fan Girl (Jadaone, 2020). Sa paggamit nito ng mall show bilang instrumentong makapagpapatagpo 
ng fan at ng idolo, inilulugar si Jane sa mas malawak na fandom na may paggalang sa anumang uri ng mapagpalayang ekspresyon ng kababaihang paghanga. Sa loob ng komunidad na ito, ipinagdiriwang ang kapangyarihang taglay ng kolektibong pagsigaw ng kababaihan at tinatanggap ang anumang pisikalisasyon ng pagsuportang umuugat sa hangaring mapansin ng iniidolo mula sa simpleng pagkaway upang makuha ang atensiyon nito, hanggang sa pakikisiksik mailugar lang ang sarili sa posisyong pinakamalapit dito na siyang pinahihintulutan pa rin ng espasyo. Sa mga sandaling ito ng pelikula, ipinapabatid sa mga manonood na may ilan ding kapuwa fan si Jane na maaaring ituring bilang komunidad na binubuo ng mga indibidwal na may parehong antas ng paghanga sa idolo na inihuhudyat ng iba't ibang paraan ng kanilang pagsuporta. Nariyan ang pagkuha ng video ng kabuoang programa sa layuning mapanghawakan nang mas matagal ang sandali, ang pagkompás ng mga inihandang banner at lobong binabaybay ang pangalan ng idolo sa layuning mapansin nito, at ang pagpupumilit na maagaw ang ipinamimigay na poster sa pag-asang magkaroon ng isang munting obhetong makapaguugnay ng sarili sa hinahangaan.

Katulad ng mga ekstrang nasaksihan sa pelikula (Jadaone, 2020), mahalaga para sa mga fans ang mga materyal tulad ng nakuhanang video o naagaw na poster na muling makapagpapaalala sa kanila ng saglit kasama ang idolo at komunidad. Lubos-lubos na pinanghahawakan ang mga obhetong ito sapagkat malay ang mga tagahangang matatapos ang pinakahihintay na sandali at babalik sa kaniya-kaniyang pamumuhay at responsabilidad na bagaman naiimpluwensiyahan pa rin ng idolo ay hindi na direktang nakatali dito. Sa pagtatangkang isantabi ang realidad na ito na siyang bahagi ng kamalayan ng mga fangirl, iginigiya ng pelikula ang sarili bilang isang kultural na materyal na muling kikiling at lalo pang makapagpapakapal sa representasyong patolohiko ng mga babaeng tagahanga. Kasunod ng pagpapakita sa personal na pagpapasya ni Jane na hindi piniling bumuklod sa kapuwa tagahanga na kumikilos batay sa dikta ng espasyong nababarikadahan at nahaharangan ng seguridad, napatitingkad nito ang maling nosyon ng paghangang nauunawaan bilang akto ng pagbuntot sa idolo. Nang piliin ng karakter na isilid nang patago ang sarili sa pick-up truck ng hinahangaan sa pag-asang matupad ang hangaring mapalapit dito, tinalikuran ni Jane ang komunidad na isa sanang mahalagang espasyo na makapagpapangalaga ng kaniyang identidad bilang tagahanga at bilang isang babae-isang pagsasawalambahala sa bisa ng komunidad na magpapaloob sa kaniya sa sunod-sunod na sitwasyong mauuwi sa kongklusyon na "never meet your hero" na tagline ng pelikula (Jadaone, 2020).

Kung babalingan ang bisa ng komunidad, masasabing may makinarya ito upang matagumpay na maibuklod ang isang indibidwal na fangirl sa 
mas malawak na fandom (Jenkins, 2018, p. 44). Nailulunsad ang pagbuo ng kolektibo sa sabay-sabay na pagigiit ng ahensiya ng mga indibidwal na kababaihang pumipili kung kanino ibabaling ang pagmamahal bilang isang tagasuporta. Katulad ng dikta sa loob ng jeepney bilang isang espasyong namamalayan ni Jane bilang isang fangirl, may dinamika ring sinasandigan at nirereprodyus ang fandom. Dahil may mga alituntunin ang espasyo (Hjarpe, 2008, para. 8), na natutulad sa konseptuwalisasyon ni Bourdieu ng field na may nabubuong kaugalian (habitus) ang paulit-ulit na interaksiyon at pakikisangkot (Bourdieu, 1984, p. 101), may mga gawi at praktis na ipinagmamalaki at ipinagbabawal sa loob ng fandom. Bahagi ng makinaryang nagpapabatid kung aling kaugalian ang tinatanggap ng fandom sa alin ang hindi ang pag-iral ng grupo ng mas matagal nang tagahanga na sistematikong naglalatag at nagbabahagi ng kanilang mga kaalaman
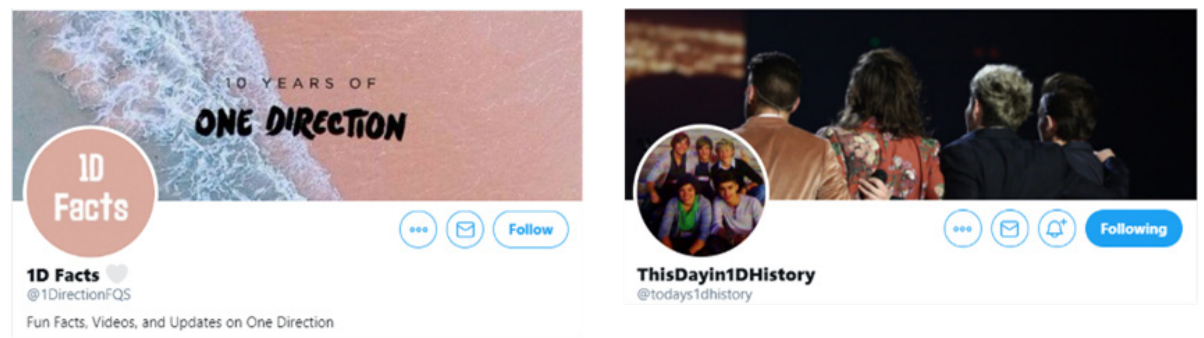

"Lina ${ }^{28}$ 's Nobody is listening

Q91CHERRYOTB

Here's a thread of larries you should follow:

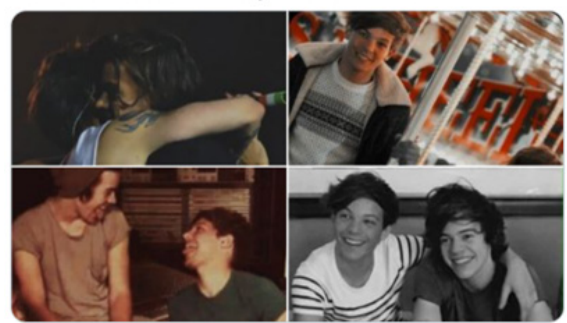

(2) Swati ${ }^{28}$ is missing Louis

A thread of masterposts for all new Larries/Twarries/Antis.

6:36 PM · Dec 8, 2020 - Twitter for Android

665 Retweets 207 Quote Tweets 3.3K Likes

3:50 AM - Jan 14, 2021 - Twitter Web App

138 Retweets 3 Quote Tweets $1.3 \mathrm{~K}$ Likes

Larawan 2. Ilan sa matuturing na fan curators sa espasyo ng Twitter partikular na sa One Direction fandom at sa sub-fandom nitong inaako ang pagkakakilanlang "Larries" na sumusuporta sa "ship" na Larry Stylinson-ang pinagtambal na pangalan ng mga miyembrong sina Louis Tomlinson at Harry Styles na pinaniniwalaang may itinatagong romantikong relasyon. Makikitang may ilang account na inihanda para sa mga bagong tagahanga upang mabatid ang ilan sa mahahalagang impormasyon (1D Facts, n.d.) at pangyayari (ThisDayin1DHistory, n.d.) hinggil sa banda. At may ilan din namang indibidwal na tweet na naglalaman ng talaan ng mga kapuwa fan na maaaring i-follow at mga thread na maaaring basahin upang maging pamilyar sa mga pangyayari at paniniwalang sinasandigan

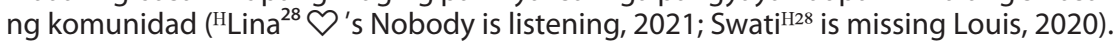


(Kompare, 2018, pp. 107-108). Nababansagan bilang "fan curators" sila ang umakko ng tungkuling gawing komportable ang mga baguhan at maipaunawa sa kanila ang dikta ng espasyo (Lar. 2) (Hjarpe, 2008, para. 4).

\section{Paglublob sa Komunidad ng Kababaihang Paghanga}

Habang unti-unting napapamilyarisa ang isang fangirl sa umiiral na dikta sa loob ng espasyong kinabibilangan, unti-unti ring lumalabo ang hanggahan sa pagitan ng mga bago at matagal nang tagahanga. Sa araw-araw na pagmamasid, pakikitungo at aktibong partisipasyon sa nabuong komunidad (Jenkins, 2018, p. 39), nalulusaw rin ang paunang bugso ng kagustuhang kumiling lamang sa idolo at napapalitan ng kagustuhang makabuo ng direktang pag-uugnayan sa mga kapuwa tagahanga. Kung gayon, mapagaalamang hindi lamang idolo ang natatanging tampulan ng pansin ng isang fan. Kaakibat ng pag-anib sa isang komunidad ang hangaring makabuo ng relasyon sa kapuwa fangirl na nangangahulugan ng pagbibigay ng oras upang palagiang makapangumusta at ng atensiyon upang lubusang makilala ang mga kabahagi ng fandom (Cook \& Hynes, 2014, p. 89)—mga pag-uugnayang inilulunsad man ng pagkakaroon ng isang idolo ay kayang humantong sa pagkakaibigang lalampasan ito (Lar. 3).
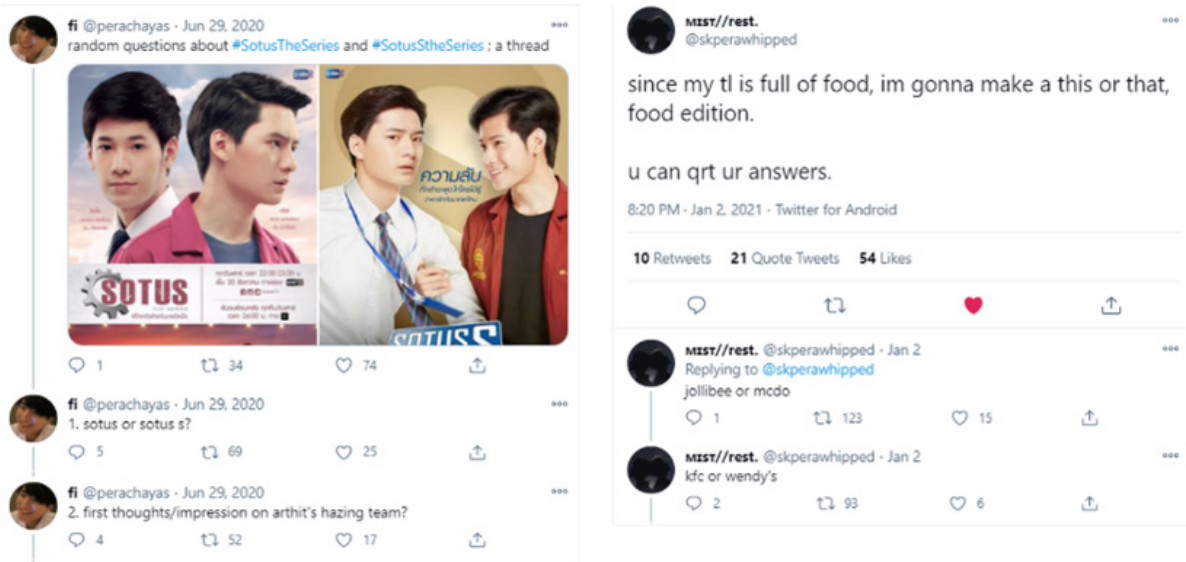

Larawan 3. Napapahintulutan ng espasyo ang mga paraang posibleng makapagpalalim ng pagkakaibigang birtuwal. Mula sa feature nitong quote tweet at reply, maaaring sumagot ang mga interesadong fan sa mga tanong na inihain ng kapuwa tagahanga na maaaring direktang kaugnay ng materyal na obhetong hinahangaan ng fandom tulad ng Thai BL Series na SOTUS (fi, 2020a, 2020b, 2020c) o kaya naman mga maliit na detalyeng labas na sa usapin ng hinahangaan (MIST// rest, 2021a, 2021b, 2021c).

Masalimuot na diskurso ang nabubuksan ng pagkilala sa fandom bilang lunsaran ng pagkakaibigan sa pagitan ng mga fangirl. Bukod sa napatitingkad nito ang mabuting dulot ng pag-akap sa identidad na tagahanga na inuunawa ng larang ng Fan Studies bilang motibasyong nakatuon sa 
usapin ng "belongingness," nagtataglay rin ito ng representasyong umiiwas sa pagkakakahon sa mga fangirl bilang mga kababaihang humalíng na humalíng sa idolo na hindi nagdadalawang-isip na gumagawa ng mga aktong ikapapahamak ng sarili para lang maisakatuparan ang hangaring mapalapit dito tulad ng pananatili ng karakter ni Jane sa poder ni Paulo Avelino sa likod kuwestiyonable nitong pamumuhay na ipinakita sa pelikula (Jadaone, 2020). Ang mismong akto ng pakikiisa sa komunidad ay maaari ding maitawid sa diskursong tumatalikod sa malawakang pagsasaklaw sa fans sa kategoryang babae at sa mga kaakibat na pagtanaw rito. Bagaman mailalarawan gamit ang terminong "homosociality" ni Eve Kosofsky Sedgwick (1994) na nagtataglay ng posibilidad na makabuo ang mga fan ng espesyal na pagkakaibigang hindi pinagagana ng romantikong paghahangad (pp. 114-115), may mga pagkakataong pinapalabo ito ng mismong retorikang ginagamit ng mga ito sa pakikipag-ugnayan sa isa't isa. Sa loob ng establisado nang pag-uugnayan, mababakás ang mga tendensiyang lantarang ipinapahayag ng isang fan ang pagmamahal nito sa isa pa na maaring kilalanin bilang isang queer expression na lumulusaw sa masaklaw na paglalakip ng kategoryang "girl" sa "fangirl." Sa pagpapalagay na babae ang halos lahat ng umookupa sa espasyo, kasabay ang katotohanang nasasaksihan sa hanay nito ang mga pagpapahayag na mailalarawan bilang queer, kinikilala ni Mizoguchi (2008) ang pag-iral ng mga "virtual lesbian" (p. 339) na nakapagsisiwalat ng mga homoseksuwal na
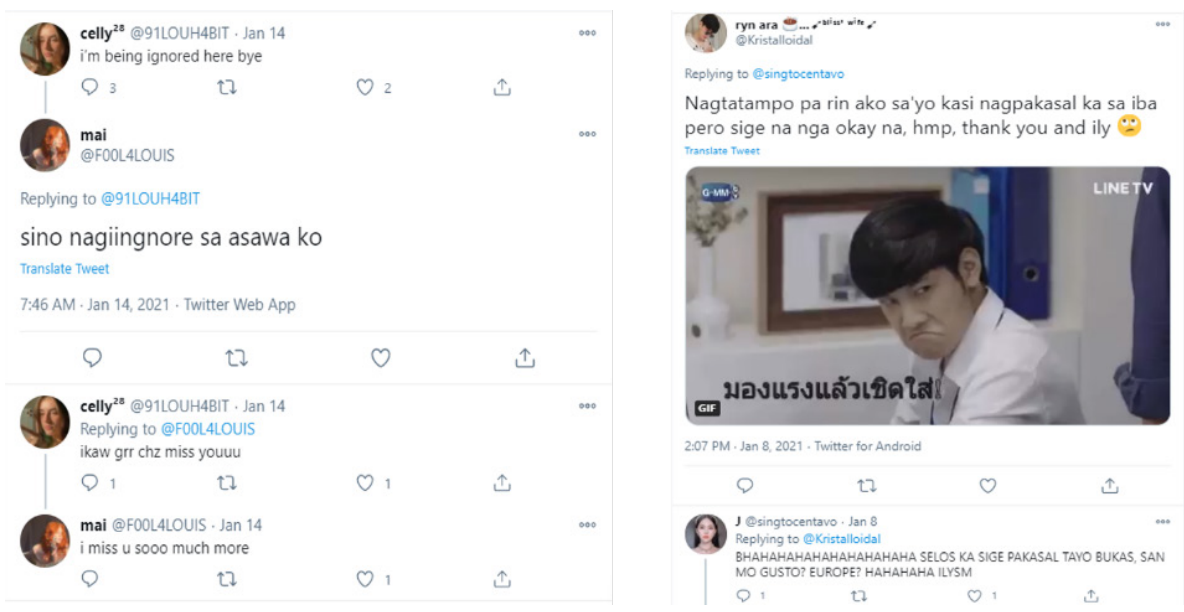

Larawan 4. Ilan sa mga partikular na halimbawang kinikilala ni Mizogushi bilang "virtual lesbians" sa hanay ng mga Larries, tagahanga ng tambalang Larry Stylinson ng One Direction (celly ${ }^{29}$, 2021a, 2021b; mai, 2021a, 2021b), at Peraya, tagahanga ng tambalang Krist Perawat at Singto Prachaya na gumanap bilang Arthit at Kongpob sa Thai BL Series na SOTUS (ryn ara, 2021; J., 2021). Interesante ang makakasariang pagsipat sa naturang fandom sapagkat kailangang isaalang-alang ang suson-suson nilang pagtalikod sa heteronormatibo mula sa pagiging tagahanga ng maituturing na male loveteam, hanggang sa pagpapahayag ng mga ganitong uri ng sentimiyento sa kapuwa tagahanga. 
tendensiya sa pamamagitan ng gawaing nakasandig sa akto ng role playing (Lar. 4).

Maraming salik ang nakapagpapakomplika sa tanong kung natuturol ba ng tekstuwal na ebidensiyang mababakasán ng tendensiyang queer ang aktuwal na seksuwalidad ng mga tagahanga. Bukod sa pinapangalagaan ng espasyo ang anonymity ng bawat isang kasapi, nagtatagpo-tagpo rin dito ang lahat ng uri ng indibidwal mula sa iba't ibang lahi, relihiyon, at henerasyon na umaakap sa malawak ding pag-unawa sa kasarian at seksuwalidad na sinasaklaw ng terminong "infinite diversity" ni alias The Fan Meta Reader (2015, para. 13-14). Sa isang banda, tulad ng nababanggit na role-play ni Mizaguchi (2008), maaari din namang sabihing paglalaro lamang ito sa pananaw ng mga tagahanga-na hindi maitutumbas ang paggamit ng mga salitang "asawa ko" o kaya naman "mahal ko" sa pagiging homoseksuwal ng mga ito. Subalit, mauuwi din naman sa kulang na pagsipat ang agarang pagbalewala sa mga naturang tendensiya bilang larolaro o biru-biruan lamang. Tulad ng pagtataya ni Lucy Irene Baker (2017) sa kaniyang pananaliksik hinggil sa mga tagahanga ng gender creative fan work, ${ }^{2}$ mababakás sa mga kinikilingang anyo o obheto ng mga fans ang kanilang paggigiit ng pansariling kasarian at seksuwalidad na lumalampas sa heteronormatibong pagpapalagay kung mabibigyan lamang ng pagkakataong isiwalat ito (para. 0.1). Sa kaniyang sarbey, lumalabas na maski madalas kilalanin ang fandom bilang espasyong pangkababaihan, may sapat pa ring panghahawak ang mga indibidwal na tagahanga sa retorikang babasag sa heteronormatibong dikotomiya ng babae at lalaki na makapagpapahayag ng kanilang pag-alagwa sa iisang nosyon ng "pagkababae" (Baker, 2017, para. 6).

Tulad ng maraming lente na maaaring gamitin upang suriin ang pagtanaw ng fans sa usapin ng kasarian at seksuwalidad, maramihan at masalimuot din ang pag-unawa ng fandom sa mga tungkuling kaakibat ng pagyakap sa identidad bilang isang fangirl. Gaano man kasalimuot, masasabing nagkakasundo pa rin ang komunidad sa pagpapamalas ng suporta sa idolo na ipinapares sa hangaring makilala at mapalapit dito bilang sukli sa lahat ng atensiyong ginugol. Dinidiskurso sa pelikulang Fan Girl (Jadaone, 2020) ang nosyon ng pagkakakilala at pagkakalapit sa idolo sa pinakaliteral na paraan na winawasak ang intrinsikong guwang na namamagitan sa isang idolo at fan. Pinatatamasa sa fangirl na si Jane ang pagkakataong mapalapit sa idolo sa pagsasara ng pisikal na distansiyang nabibiswalisa sa napakapisikal ding pag-uugnayan na mabubuo sa pagitan ng dalawa-pisikal na paghahati ng iisang espasyo; pisikal na paghahati ng alak, sigarilyo at droga; at maging ang pisikal na paglalapit ng mga katawan sa pinakarurok na akto ng pagtatalik. Ang lahat ng ito ay unti-unting mauuwi sa pisikal ding akto 
ng pagmamaniobra at pang-aabuso ng idolo sa tagahanga na wawakasan sa pisikal ding pag-igpaw ng dalaga sa pagpapatihulog ng sarili mula sa umaandar nitong sasakyan.

Nalalayo sa representasyong itinatanghal ng pelikula hinggil sa paraan kung paano matatamasa ng isang tagahanga ang hangarin nito, may alternatibong artikulasyon ang fandom sa pagbuo ng malapit na relasyon sa idolo nang hindi pinanghihimasukan ang personal at pribadong espasyo ng kapuwa kasangkot. Sa konteksto ng birtuwal na pagbabad sa loob ng isang fandom, kagyat na maipagpapalagay na ligtas ang pamamaraang inihahain sa isang fangirl sapagkat hindi nito isinasangkot ang aktuwal na katawan ng fan. Hindi tulad sa pagsasakasangkapan ng pelikula sa batang katawan ni Jane na ipinaloob sa sunod-sunod na panganib-panganib ng patagong pagbuntot sa idolo nang mag-isa, panganib ng kakaharaping panggagalaiti sa puntong madiskubre ng aktor, at panganib ng sasambulat na katotohanan na ang hinahangaan ay natutulad sa ama-amahang abusado na pilit niyang tinatakasan, pananggalang ng mga fan sa espasyo ang bisa ng anonimidad na nagtatago ng kanilang pisikal na pagkakakilanlan mula sa kanilang tunay na pangalan hanggang sa kanilang hitsura (Trinidad, 2018, pp. 159-160; TWC Editor, 2010, para. 1.2). Ang mga account na ito, na walang bakás ng kanilang tunay na pagkatao, ang ginagamit sa pakikipag-ugnayan sa mga idolong may account din sa espasyong online. Bukod sa pagtugon sa mga tweet ng idolo, madalas din sumailalim ang mga fan sa tinatawag na "tweetspree"- o panaka-nakang pag-tag at pag-iiwan ng mensahe sa mga hinahangaan upang makuha ang "notice" nito-retorika ng espasyo na pumapalit sa pisikal na pansing maaaring idulot ng idolo sa tagahanga sa pamamagitan ng pag-iiwan ng "heart" o reply sa tweet nito. Ilang halimbawa ang mga ito sa pagtatagpong hindi man tumatawid sa mundong pisikal ay maituturing pa rin bilang direktang interaksiyon sa pagitan ng fan at idolo na may kakayahang makapaglunsad ng "air of extemporary intimacy" (Muntean \& Petersen, 2010, para. 25).

Bagaman ipinagpapalagay na malaking bahagi ng komunidad ang yumayakap ng praktika ng fangirling na posibleng maisagawa sa espasyong birtuwal, may paglalatag pa ring isinasagawa ang fandom sa mga mangangahas na buwagin ang pisikal na guwang sa pagitan ng idolo at fan. Para sa mga pilit iginigiit ang pisikalisasyon ng hangaring mapalapit sa idolo sa pamamagitan ng stalking-isang aktong matagal nang ikinakabit sa mga tagahangang humalíng na humalíng, ipinapaalala ng pagbabad sa komunidad at ng karampatang sosyalisasyon sa loob nito na hindi kailanman sagot ng modernong fandom ang pangungunsinti sa lihim na pagbuntot sa idolo. Sa kolektibong pagyakap ng tunguhing unahin ang kaligtasan ng hinahangaan, may makinarya ang fandom na didisiplina at 
pupulis sa mga akto ng paghangang lumalampas sa hanggahan ng pagiging katangap-tangap. Sa mga pagkakataong ito, kumakasangkapan ang fandom ng mga konseptong boundary, dignity, at privacy bilang karapatan ng bawat indibidwal, tanyag man o hindi (Lar. 5).
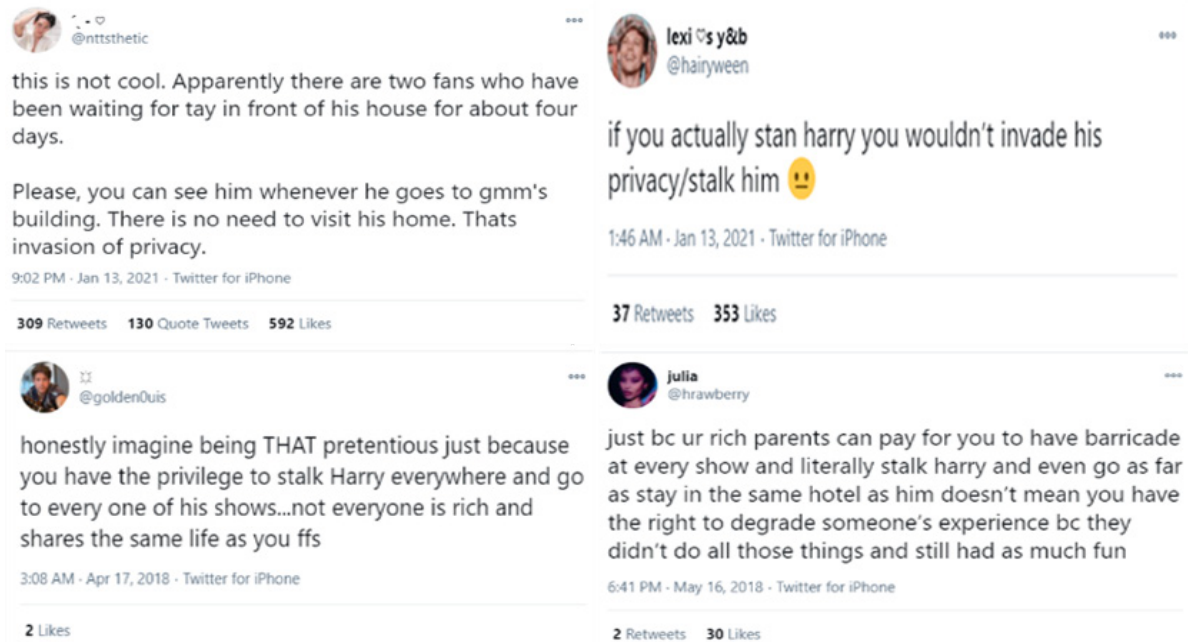

Larawan 5. Sa parehong Thai BL fandom at One Direction fandom na pinag-uugatan ng empirikong datos ng papel, makikita na nagkakasundo ang mga fan na bahagi ng pagiging isang tagahanga ang pangangalaga sa karapatang maging pribado ng idolo. Bukod dito, naniniwala rin ang komunidad na may mga akmang lugar na maaaring pisikal na katagpuin ang mga idolo katulad ng mall show sa kaso ng pelikulang Fan Girl (Jadaone, 2020) at ang nababanggit 'gmm's building' sa screenshot ng Tweet ni ', - $D$ [@nttsthetic] (2021). Sa mga hinangong halimbawa, interesante na sabay ring natatapik ng pagpuna sa akto ng stalking (Lexi $\oslash$ s y\&b, 2021) ang mga salik na nakakapagpahintulot nito tulad ng kinabibilangang uri ng mga tagahangang lumalabag ( [@golden0ius], 2018). Bukod sa lantarang usapin hinggil sa yaman, maipagpapalagay na malay rin ang mga tagahanga sa pagkakaiba-iba ng kanilang mga personal na kakayahan, responsabilidad, at pamumuhay sa pang-araw-araw (Julia, 2018).

Sa pagiging proactive sa halip na reactive ng fandom, masasabing hindi natatapos sa pamumulis o panenermon ang kanilang solusyon upang kaharapin ang suliranin ng stalking. May mga alternatibo itong paraan ng paghangang gagagad sa katuparan ng pangarap na mapalapit sa idolo. Mga anyong naghahain ng rendang pipigil sa tuluyang pagkabanat ng hangaring madalas tumungo sa destruktibong akto ng stalking; at mga praktikang maitutumbas sa "ways of operating" ni De Certeau (1984, p. xx) na sa pamamagitan ng "segundaryong produksiyon" (pp. xv-xx) ay malikhaing nagagamit ng mga tagahanga ang mga available na bagay upang maiakma sa interes at dinamika ng kanilang komunidad.

Dahil nakasandig ang hangaring mapalapit sa idolo sa pagiging nariyan (present) nito na hindi laging posible dahil malay din ang komunidad na may 
buhay ang idolong hiwalay sa pagiging celebrity, may mga apropriyasyon at reapropriyasyong isinasagawa ang fandom upang mapanatiling "buháy" kahit ang imahen man lang ng idolo. Sa pag-iimbak ng mga "artifacts" na nakalagak sa kanilang kolektibong alaala, kaakibat ng pagiging accessible ng mga ito, nabibigyan ng kagyat na kakayahan ang mga fans-bilang indibidwal man o kolektibo, na lumikha, muling lumikha, mag-curate at magpamudmod ng mga materyal sa loob ng mismong komunidad (Jenkins, 2018, pp. 44-45). Dahil dulot ng pakikiisa sa fandom ang pagkakaroon ng sapat na kaalaman hinggil sa canon-o yaong mga detalye, impormasyon at pangyayaring umuugat mismo sa totoong pag-iral ng idolo, napatutunayan ng tradisyon ang praktikang nabuo ng komunidad sa ilang taon nitong pagiral ang pagkapit nito sa hindi mabilang na "microbe-like operations" (De Certeau, 1984, xiv-xv) na kalat-kalat at sapin-sapin (Jenkins, 2018, p. 42).

Halimbawa, natutugunan ang hangaring makabuo ng matalik na pagkilala sa idolo kahit wala ang pisikal nitong presensiya sa pagtitipon ng mga kultural na materyal o pagpapahalagang malapit dito. Bukod sa kinikilala ang idolo bilang suhetong hindi lamang isang celebrity, kundi isa ring indibidwal na may sariling "taste" at libangan, binubuksan nito ang lagusan na makahanap ang mga tagahanga ng iba pang anyong kultural
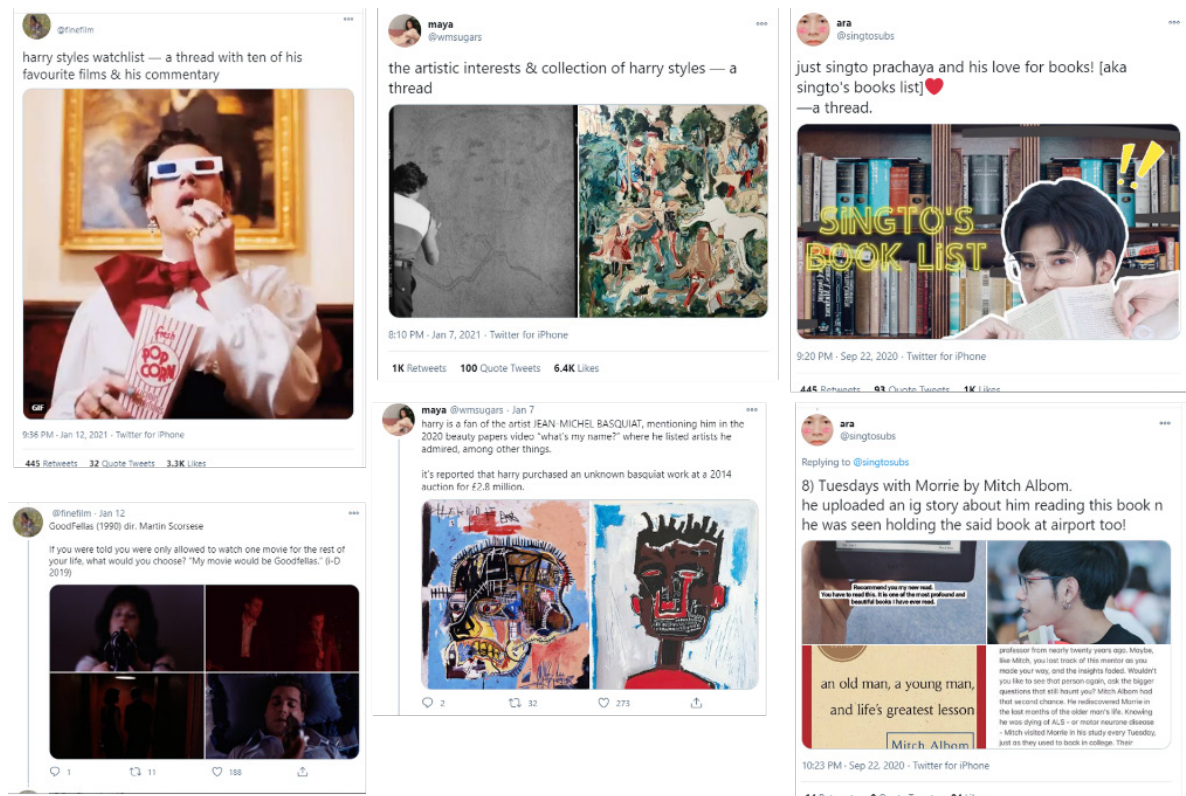

10223 PM- Sep 222020 - Twiter for iphone

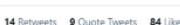

Larawan 6. llang talaang tinipon ng ilang tagahanga na nagpapakita ng mga kultural na gawaing kinahihiligan ng kanilang mga idolo (@finefilm, 2021a, 2021b; maya, 2021a, 2021b; ara, 2020a, 2020b) . 
tulad ng mga pelikula, likhang-sining, at akda, at makapulot ng pagkatuto mula sa mga ito (Lar. 6).

Kung itinuturing ni Jenkins (2018) na "generative" ang mga gawaing ito na nakapaglulunsad ng panaka-nakang diskurso sa komunidad (pp. 50), epektibo rin namang nakapagsisimula ng aliw at pag-uusap sa mga tagahanga ng transnasyonal na anyong pangmidya ang metaporikal na pagtatangkang ilapit ang idolo sa sariling kultura. Sa mga kasong ito, imahinatibong inilulugar sa mga pamilyar na anyong kultural ang mga idolo na nagagawang mapagtagpo sa pamamagitan ng mapaglarong pag-uugnay ng mga detalye ng sariling kultura (halimbawa, pagdaraos ng isang Jollibee birthday party at pamilyaridad sa pelikulang She's Dating the Gangster ng KathNiel) sa imahen ng mga idolong hayag na nalalayo sa aspektong pisikal at kultural sa Filipino nitong tagahanga (Lar. 7).

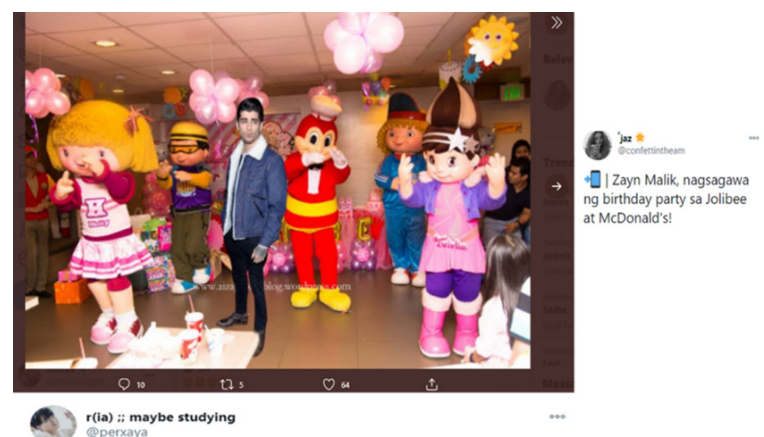

Larawan 7. Kinikilala ng larang ng Fan Studies bilang "Fandom at Play," pilit na inuungkat ng mga tagahanga ang piling representasyong kultural ng bansa sa hangaring ilapit dito ang mga idolo (Jaz is listening, 2021; r(ia) \# maybe studying, 2021).

She's dating the gangster but it's KS

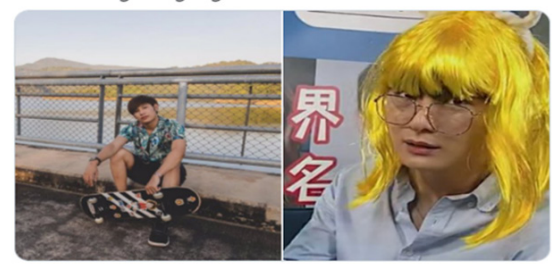

11:26 PM - Jan 11, 2021 - Twitter Web App

12 Retweets 6 Quote Tweets 57 Likes

Sa patong-patong at sala-salabid na paraan ng paglikha upang makisangkot, sabay na naipapamalas ng komunidad ang malikhaing kapasidad at intelektuwal na dunong. Sa daling pagtagpi-tagpiin ang mga umiiral nang impormasyon, at sa palagiang pagpapamalas ng abilidad na magbalik-tanaw upang iugnay ang mga naganap na sa nagaganap sa kasalukuyan o upang pagtagpuin ang mga tematikong pagkakatulad ng iniidolo sa iba pang kultural na anyo, napapasubalian ng fandom ang paniniwalang emosyon lamang ang puwersang nagpapagana ng kababaihang paghanga. Sa pamamagitan ng maituturing na maliliit na halimbawa ng 
fan labor, napagtatagpo ng mga tagahanga ang "analytical mind" at ang "desiring body" sa kanilang paglikha ng isang "total female subjectivity" (Coppa, 2008, para. 2.19).

Kung gayon, hindi maihahanay ang litanya ng pagpapakitang-gilas ni Jane bilang marubdob na tagahanga ni Avelino-pamilyaridad sa kompletong birthday at astrological sign nito, pangalan ng mga inaalagaang aso, unang palabas sa parehong network, at iba pa (Jadaone, 2020) bilang sobra-sobrang kultibasyon ng walang katuturang kaalaman o pagpapahalaga sa walang kabuluhang kultural na materyal (Jenkins, 1992, p.10) kung naisapakete ang mga ito bilang impormasyong paglulunsaran ng transpormatibong reproduksiyon na palagiang pinapagana ng komunidad at ng pakikibuklod dito. Sa pagkilala sa kakayahan ng fandom tungo sa transpormatibong paglikha, hindi lamang nawawaksi ang pananaw na nanatiling masigasig na tagakonsumo lamang ang mga tagahanga, kundi naisusulong din ang maingat nilang pagsasakatuparan ng hangaring mapalapit sa iniidolo. Halimbawa, sa pamamagitan ng paglikha ng fan artpisikal man tulad ng collage na nakita ni Jane habang nagtatago sa likod ng pick-up truck ni Avelino (Jadaone, 2020) o digital tulad ng madalas ipinaabot sa idolo sa pamamagitan ng pagta-tag sa personal na account nito sa Twitter-naiaangat ng isang indibidwal na tagahanga ang sarili sa malawak na komunidad at nabibigyan ng pagkakataong makatanggap ng pagkilala o atensiyon, gaano man kasaglit, mula sa idolo.

Ang pagsandig sa kapasidad na lumikha upang ilapit ang sarili sa idolo ay natutugunan din ng malawak at establisado nang mundo ng fan fiction. Anupaman ang anyo-naratibong nahahawig sa isang buong nobela, one-shot o single-chaptered alternative universe na maituturing bilang maikling kuwento, self-insert fan fiction, blurbs na pinagkakasiya sa loob ng 250 na salitang pinahihintulutan sa isang tweet, o social media AU na kumakasangkapan ng parehong salita at imahen-tumatapik sa intelektuwal na pagpapamalas ng mga tagahanga ang walang humpay na pagbuo ng mga naratibo at senaryong libreng ipinapamahagi sa buong komunidad. Gayumpaman, umiiral din ang kritisismong hindi isang intelektuwal na gawain ang pagsusulat at pagbabasa ng fan fiction dahil sa likas nitong pag-uugat ng mga hangaring seksuwal ng mga kababaihang tagahanga (Larsen \& Zubernis, 2013, p. 4). Mula sa tago at hayag, kumbensiyonal at hindi na mga aktong seksuwal na isinasagawa ng mga karakter na hango sa idolo o mga iniidolo na nagtataglay ng iba't iba at pinaglalabo-labong kasarian at seksuwalidad na naakses sa fan fiction (Meggers, 2013, p. 58), nagiging alternatibong espasyo ang mga naratibo upang magalugad ng mga kababaihang tagahanga ang kanilang seksuwalidad nang hindi inilalantad 
ang mismong pisikal na katawan sa anumang kapahamakan o negatibong epekto (Larsen \& Zubernis, 2013, p. 4).

Bukod sa pagkatuto at pagkakilala sa lawak ng sexual identity na maaaring kilalanin para sa sarili, sityo rin ang fan fiction ng pagsulong ng progresibong pagtanaw sa kasarian. Sa "slash" bilang halimbawang genre na romantikong (at kung minsan erotikong) pinagpapares ang dalawang orihinal na lalaking karakter, napagninilayan kung papaanong maaaring ibatay ang romansa sa higit na pantay na pagtingin sa kasarian na kahit ang karakter na lalaki ay naghahangad ng intimacy at nagpapamalas ng vulnerability (Zsila \& Demetrovics, 2017, p. 4; Jenkins, 2018, p. 44; Scott, 2019, p. 30). Sa kasalukuyan, nauuso rin sa espasyong online ang muling pagbaling sa youth at girls' culture na pagtatago ng mga diary at pagbuo ng mga collage at scrapbook upang makapahain ng muling naratibisasyon at biswalisasyon ng mga sandaling mahalaga para sa mga idolo [Lar. 8]. Bukod sa napagtitibay nito ang pagkilala sa mga kalalakihan bilang yumayakap sa mga bulnerableng emosyon na bahagi ng malawak na proyektong punahin ang patriyarkal na kaayusan (Baker, 2017, para.3) — na sa isang banda ay tinangka ring ipaloob sa pelikulang Fan Girl (Jadaone, 2020) nang ipinakitang bumuhos ang emosyon ni Avelino na idinulot ng matagal na kinimkim na guilt sa di-pagbisita sa ina sa ospital habang nabubuhay pa ito at sa di rin pagbuo ng maipagmamalaking pagtrato sa kaniyang mag-ina-inihahain din ng mga naratibong tulad nito ang boses ng kababaihang nagsusulong na alternatibong pagkalalaki na maaaring kumilates sa hegemonikong maskulinidad (Braid, 2014, p. 75).
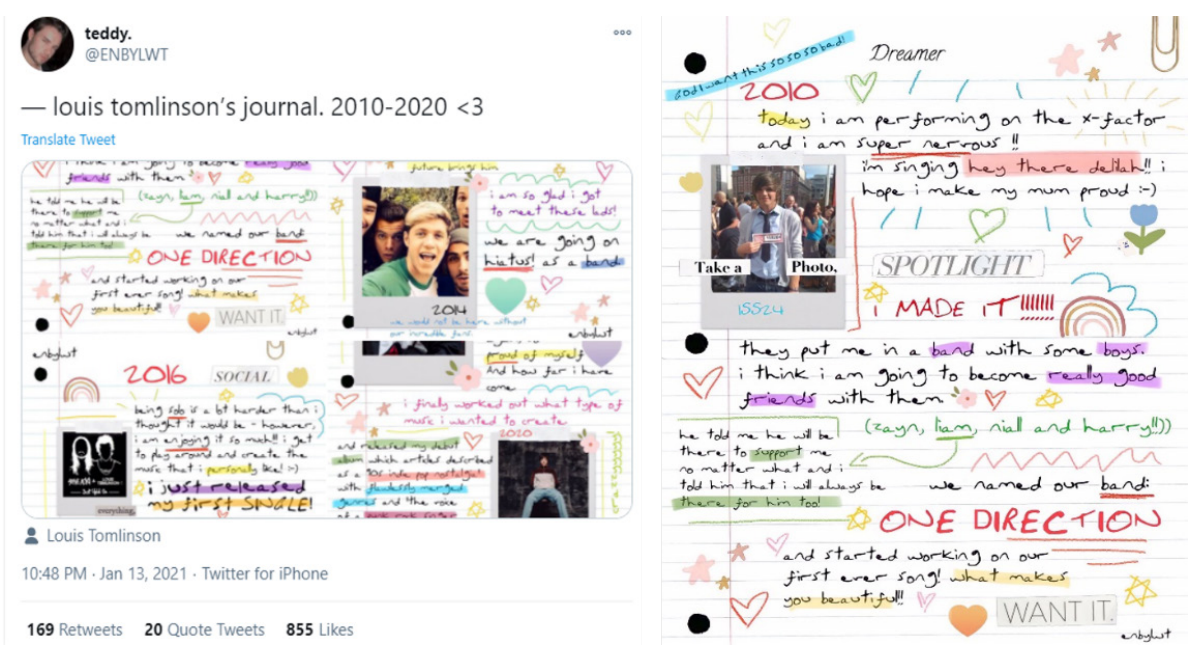

Larawan 8. Sa pamamagitan ng reimahinasyon ng mga taóng inilagi ni Louis Tomlinson sa bandang One Direction, naipapakita ng mga fan ang isang anyo ng pagkalalaking hindi mapanganib para sa mayorya ng kaniyang mga tagahanga (teddy, 2021). 
Bagaman pinupuna rin ang fan fiction, partikular na ang "slash," sa hindi nito pag-alingawngaw sa realidad at karanasan ng mga homoseksuwal (Zsila \& Demetrovics, 2017, p. 5), hindi matatawaran ang epekto nito ayon kay Heather Meggers (2013) na nakapaglulunsad ng higit na pagkilala at pagtanggap sa mga miyembro ng LGBTQ+ (pp. 61-62). Sa pagiging lantad sa mga fan work at sa interaksiyon hinggil dito ang usapin ng kasarian at seksuwalidad, nakapaglulunsad ang praktika ng pagbabasa ng fan fiction ng mas positibong perspektibo at pakikipag-ugnayan sa kapuwa tagahangang hindi sumasandig sa umiiral na gender binary (Meggers, 2013, p. 62). Kung gayon, maaaring sabihing may puwang din ng pag-aakma ang sosyalisasyong isinasagawa sa loob ng fandom sa higit na malawak na kilusang nagaganap sa labas nito (Lar. 9).

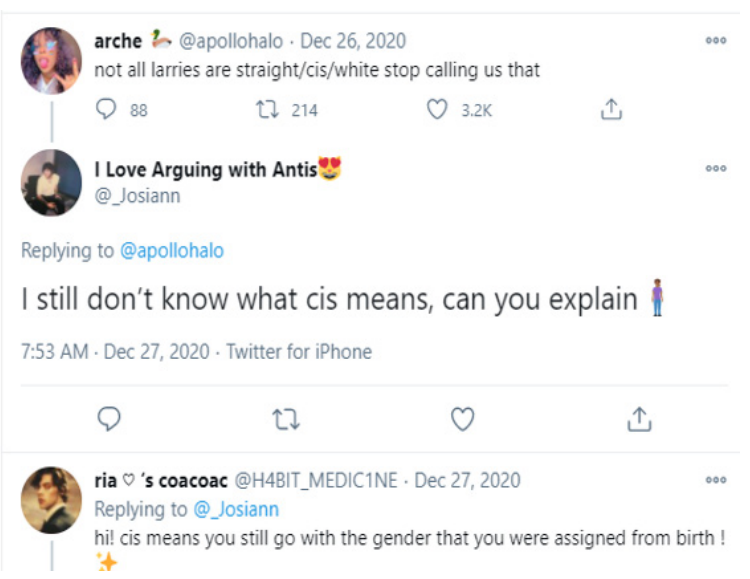

Larawan 9. Ang ganitong kamalayan hinggil sa pagiging diverse ng komunidad ay nagreresulta sa pagkatuto ng isang indibidwal na fan na magpasikot-sikot sa espasyo nang hindi kaagad ipinagpapalagay ang seksuwal na identidad (maging uri at lahi) ng kapuwa tagahanga. (arche, 2020; I Love Arguing with Antis, 2020; ria $\triangle$ 's coacoac, 2020).

Mistula mang umuugat sa pagiging transpormatibo ng fandom ang paglalarawan dito bilang progresibo at perpekto, mainam pa ring kilalanin ang kasalimuotang nasasaksihan sa espasyo. Bagaman pinakikinang ng mga nabanggit ang fandom bilang sityo ng kaluguran, mababakasan pa rin ito ng pagtatalaban dahil binubuo pa rin ito ng mga indibiwal na may kaniyakaniyang pag-uugali at paniniwala (Coscarelli, 2020, para. 8). Hindi man isahan at direkta ang ugnayan ng "toxicity" at ng fandom, may nagaganap pa ring nakalalasong pamumuna sa loob ng komunidad. Halimbawa, sa kaniyang pananaliksik hinggil sa mga fan fiction na nailimbag para sa pagkonsumong mainstream, kinuwestiyon nina Monica Flegel at Jenny Roth (2014) ang pagtanaw na femenista ang espasyo ng fan fiction na siyang sumusuporta sa kababaihang paglikha. Dahil kakikitahan ng mga diskursong laban sa babaeng manunulat-kesyo "derivative" ang mga nalilikha nitong kuwento na hindi pa nasusuportahan ng sapat na panghawak sa retorika ng panulat (p. 57-59)—napapasubalian ang masaklaw na paglalarawan sa 
genre bilang "empowering" lalo na para sa mga kababaihang inietsapuwera ng sariling komunidad dahil sa pagpapasya nitong iwanan ang fan fiction bilang "labor of love," at tumungo sa "labor economy" na mas epektibong kakikitaan ng pagiging "intellectual property" ng kanilang mga akda (p. 61).

\section{Sapagkat "Hindi na ako Bata": \\ Ang "girl" sa Fangirl at ang Paggigiit ng Higit na Ahensiya}

Bukod sa pagsulong ng tagline nitong "Never Meet your hero," pinanghahawakan din ng pelikula (Jadaone, 2020) ang katagang "hindi na ako bata" na makailang beses nasambit ng fangirl na si Jane. Sa pagsipat ng ilang piling fandom upang makapag-alok ng alternatibong karakterisasyon ng kababaihang paghanga, lumalabas na ang paggigiit ng hindi pagiging bata ay tinatahak din ng komunidad na matagal nang nasasadlak sa karakterisasyong "childish and immature" (Stanfill, 2011, para. 3.6). Maláy man o hindi, naihihiwalay ng mga fangirl sa pamamagitan ng sariling pagkilos sa kanilang espasyo ang patolohikong pagkakakilanlan. Napatutunayan nila halimbawa ang kanilang pagiging intelektuwal sa hindi kagyat na pagtanggap sa anumang inihahain para sa kanila. Hindi madaling utuin ang mga fangirl. ${ }^{3}$ Bukod sa bahagi ng kanilang praktika ang pagiging kritikal sa mga impormasyong inilalabas ng mainstream media hinggil sa hinahangaan (Jenkins, 2018, p. 44) (Lar. 10), may sapat din silang kaalaman hinggil sa pasikot-sikot ng industriyang kinabibilangan ng kanilang iniidolo na mababakás sa kanilang usap-usapan hinggil sa realidad ng marketing at PR na madalas ginagamit ang mga konseptong tulad ng "stunt," "promotion," "image build up," "media training," at "papping."

Napapasubalian din ng mga tagahanga ang paglulugar sa kanila bilang "adoring audience" na mas kinikilingan ang emosyon kaysa ang katwiran sa tuwing ipinapamalas ang balanseng paghanga at pagkabigo nito sa idolo at mga obhetong umuugat dito (Jenkins, 2014, p. 41). Mga fangirl man ang matuturing na unang depensa ng idolo sa banta ng kritisismo-tulad nang ipagtanggol ni Jane si Paulo sa pelikula laban sa aleng nag-akalang suplado ito sa pamamagitan ng pagsasabing "pagod lang" ang idolo (Jadaone, 2020), mga fan din ang unang hanay na pagmumulan ng kritisismo lalo pa't batid nilang hindi ganap na perpekto ang iniidolo. Bukod sa nabubura ang taguring "brainless consumers" (Jenkins, 1992, 10), napatitingkad ng mga fan ang hindi pagiging labis na humalíng sa pamamagitan ng pagpuna sa kakulangan halimbawa ng mga merchandise na umuugat sa idolo, maging sa pagbatikos sa mga pagkakasalang nagawa nito. Mas masalimuot kung gayon ang pagtanaw at pagturing ng mga fan sa kanilang idolo sa puntong maling isipin na bukod-tangi sa kanilang fangirling ang pagsinta rito. Katulad ng pagkakategorya ni Paulo sa fan na si Jane bilang "nanay" nang 
magbunganga ito sa loob ng kotse tungkol sa inaakalang pambababae sa isang maybahay (Jadaone, 2020), ikinagagalit ng mga fans ang pagkakamali ng idolo at sinusubukan ding disiplinahin ang mga ito (Lar. 11).

Hindi nagwawakas sa pagiging mabagsik ang pagpapamalas ng mga tagahanga ng kanilang kritikalidad na umuugat sa pagkilala sa idolo bilang hindi perpektong indibidwal. Itinatawid din nila sa malikhaing paglalaro ang pagpapatibay na ang kanilang paghanga ay hindi lamang dulot ng mababaw
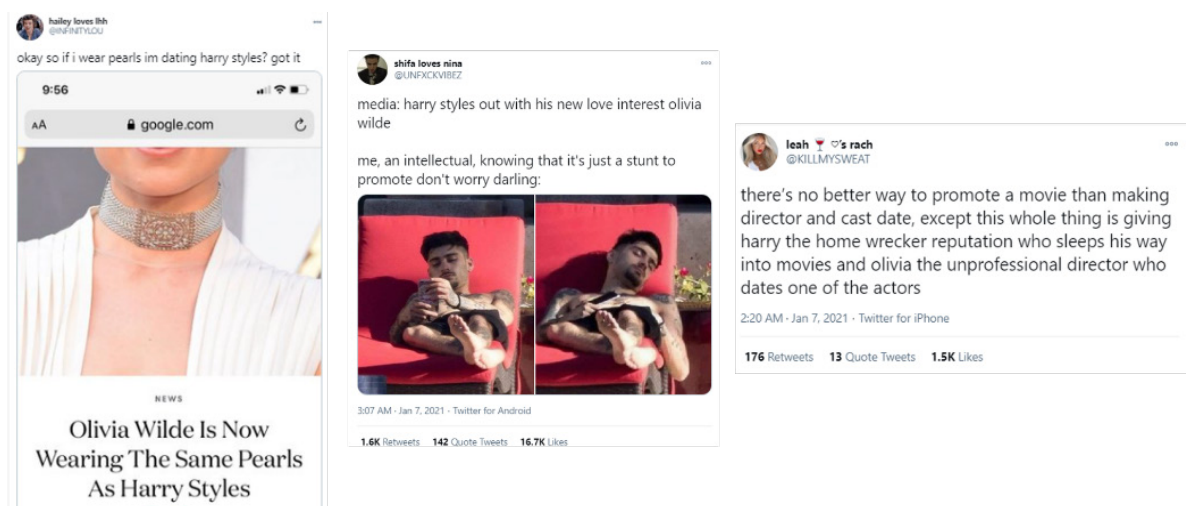

Larawan 10. Hindi katulad ng agarang paniniwala ni Jane sa kakisigang ipinakita ng idolong si Avelino sa Gandang Gabi Vice guesting nito na nagkaroon ng pag-aming tunay niyang mahal ang isang babae sa tuwing isinasakatuparan ang gusto nito (Jadaone, 2020), may iba't ibang antas ng pagtutol na sinusuong ang mga fangirl. Mula sa lantarang pagsiwalat ng pagiging wala sa katwiran ng mga lumalabas na headline hinggil sa idolo (hailey loves Ihh, 2021), lantaran ding ginagamit ang salitang "intelektuwal" sa hindi agarang pagkagat sa anumang pinapakain sa kanila ng midya (shifa loves nina, 2021), at mabusisi ring naghahain ng paliwanag na nakapagsisiwalat sa matagal nang estratehiyang pinanghahawakan ng industriya (leah D's rach, 2021).

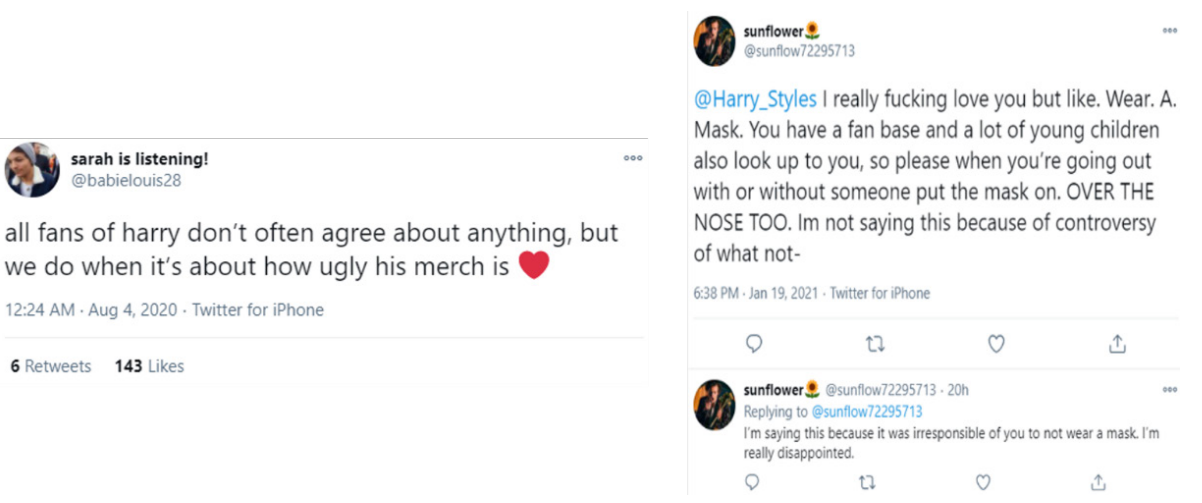

Larawan 11. Indikasyong pinapagana ng mga fangirl ang kanilang intelektuwal na kakayahan sa pagiging bukás sa katotohanang hindi perpekto ang idolo (sarah is listening!, 2020; sunflower, 2021). 
na dahilang dinidikta ng kanilang pisikal na atraksiyon. Sa pamamagitan ng praktikang tinatawag bilang "idol roasting" na siyang ginagamit ng mga tagahanga upang patingkarin ang maliit at di-seryosong kahinaan ng idolo, nagkakaroon ng sandali sa loob ng fandom na sabay na itinatampok at pinagtatawanan pa nga ang katayuang hindi perpekto ng tampulan ng paghanga (Lar. 12).
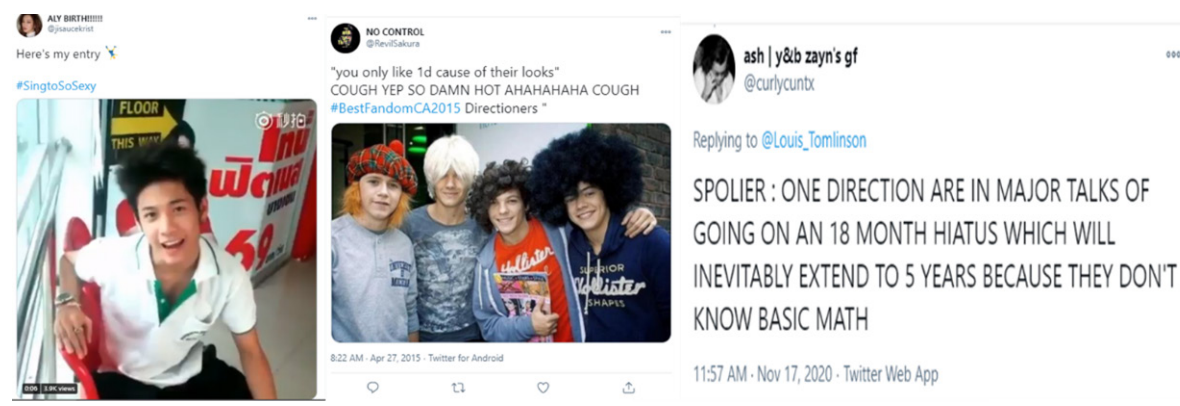

Larawan 12: Halimbawa ng idol roasting na nagaganap sa loob ng fandom na inilalantad nang direkta ang mga sandaling hindi kaguwapuhan ang mga idolo (ALY BIRTH, 2021; NO CONTROL, 2015) o kaya naman ay pinagtutuunan ng pansin ang mga pagkakataong nagpamalas ito ng kakulangan (ash | y\&b zayn's gf, 2020) na natutuldukan ang imaheng labis na humalíng ng mga fangirl.

Nakatutuwang naisasaimahen din ang hindi pagiging perpekto ng mga idolo sa paglulugar nito sa mga naratibong piksiyonal na tinatawag na "crack." Sa genre na ito ng fan work, nabubura ang pagiging nakakikilig na leading man ng iniidolo, at inilulugar sila sa mga senaryong gagalugad sa mga aspekto ng kanilang katangian at pagkatao na sadyang hindi perpekto (Lar. 13).

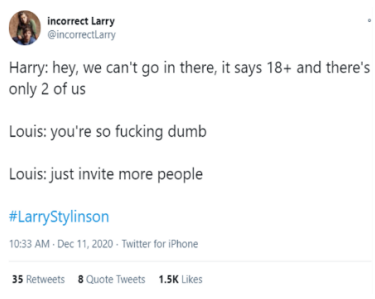

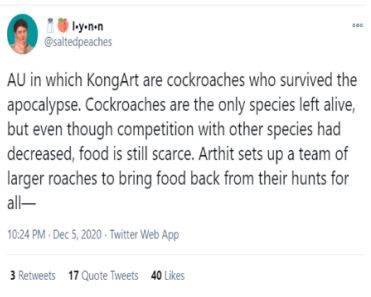

Larawan 13. Mga pilyong likhang-sining na naglulugar sa mga idolo sa posisyong katawa-tawa (incorrect Larry, 2020; lynn, 2020), nagagalugad ng crack ang paglalarawang hindi perpekto $\mathrm{ng}$ idolo na lumalayo sa madalas na pagpapahalaga sa mga tagahanga.

Sa pamamagitan ng kanilang praktika, nakaiilag din ang mga tagahanga sa pagsasaposisyon sa kanila bilang mga indibidwal na lubog sa pantasya. Bagaman ipinapamalas pa rin ang panaka-nakang hangarin na mapalapit sa idolo sa paraang romantiko katulad ng pagpapamalas sa pelikula kung paano hinuhubog ng imahinasyon ni Jane ang mala-leading man na idolong gagamot sa kaniyang sugat, kukumutan ang kaniyang nilalamok na katawan, 
at maghahatid sa kaniya sa sakayan ng bus upang ligtas na makauwi (Jadaone, 2020), ang artikulasyon ng mga pagpapangarap ay hindi nangangahuluhan ng pagkalusaw ng panghahawak sa realidad. Maituturing bilang inside joke sa komunidad, nailulunsad ng pagpapangarap na naisasapakete sa nakatutuwang paraan ang biruan at asarang makapagpapabuhay ng diskurso sa loob ng espasyo-mga diskursong kumakasangkapan ng sariling salitang nabuo ng komunidad (tulad ng "clowning" at "IRL" o "in real life") upang mapanatili ang kaayusan sa loob ng fandom at hindi sumadlak nang tuluyan ang isang fan sa mundo ng pantasya (Lar. 14).

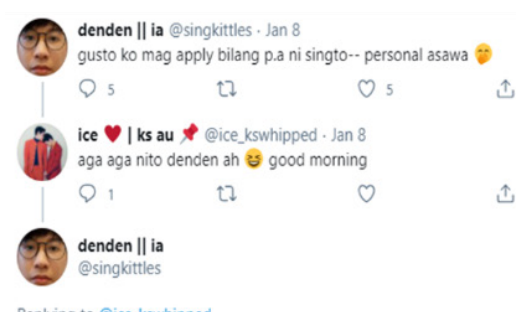

good morning ice hahahaha ganito kapag stress sa acads

Translate Tweet

10:48 AM · Jan 8, 2021 - Twitter for Android

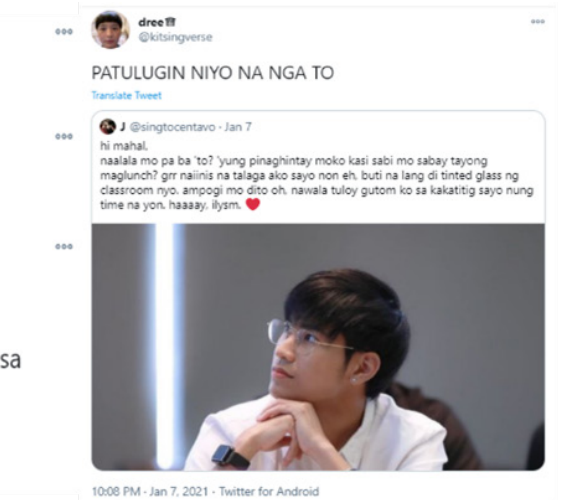

$10.00 \mathrm{PM} \cdot \operatorname{Jan} 7,2021 \cdot$ Twitter for Android

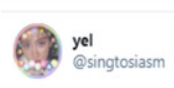

@singkittles@kitsingverse @singtocentavo good morning naman sa tatlong to na bungad at laman ng aking tl keep clowning mga bbs

Translote Tweet

$7: 24$ AM - Jan 8, 2021 - Twitter for iPhone

Larawan 14. Bilang nasa antas ng biro, matuturing na may bahid ng katotohanan ang pagpapantasyang nagaganap. Gayumpaman, masasabi ring may pagkilalang temporal lamang ang naisasagawang paghiwalay sa realidad sapagkat umiiral ang tungkulin ng kapuwa tagahangang makasasaksi ng pagpapangarap ang pagbibigay ng nakatutuwang pagtugong susupalpal dito (denden || ia, 2021a, 2021b; ice <3 | ks au, 2021; yel, 2021; dree, 2021; ilyKS, 2021).

Madali namang iniuugnay ng pelikula ang pagiging lubog sa pantasya sa pagpapakita ng libog ng mga karakter. Nailunsad ng mismong gloripikasyon ng pelikula sa eksenang nagpapakita ng ari ni Avelino na lumikha ng ingay sa social media, patong-patong ding seksuwal na akto ang ginamit nito upang sabay na isadlak si Jane bilang fangirl na lunod sa pantasyang hindi nakakikilala ng realidad at bilang dalagang mistulang naigigiit ang kaniyang hindi na pagiging bata (Jadaone, 2020). May katotohanan naman sa ganitong pagsipat ng pelikula gayong hindi maikakaila ang pagiging 
seksuwal at erotikong espasyo ng fandom. Bukod sa nagagalugad ng mga tagahanga ang kanilang mga seksuwal na identidad sa pamamagitan ng likhang-sining na nakapaghahain ng iba't ibang hangaring seksuwal, may sapat na kalayaan din ang espasyo na kinikilala ang mga kababaihan bilang mga "sexual beings" sa pamamagitan ng bukas na diskurso at eksplorasyon ng mga hangarin at pangangailangang seksuwal nang hindi ipinapaloob sa usapin ng "embarrassment, shame and guilt" (Meggers, 2013, p. 69). Ang ganitong pagkakalantad sa iba't ibang ekspresyon ng seksuwalidad ay nagiging mapagpalaya sapagkat hindi lamang ito nagiging alternatibo sa kulang na kulang na sex education sa bansa, kundi nagiging sityo rin ng pagmamalay ng isang tagahanga ng kaniyang sariling seksuwalidad sa paraang impormatibo at positibo. Kung gayon, hindi natatapos ang pagiilusyong seksuwal ng mga tagahanga sa usapin ng delusion; may natutuhog na pagkatuto ang mga ito hangga't hindi ipinapananggalang ang pisikal na katawan at hindi nalilimutang lumublob sa espasyong pangkababaihan na isang alternatibong realidad na makadaragdag sa representasyong isinagawa ng pelikula.

Sa maituturing na pinakamataas na antas ng pag-igpaw sa representasyong lulong sa pagpapantasya ang mga tagahanga, maaaring ipaloob ang malawakang pagkilala ng mga fans sa kaniya-kaniyang responsabilidad bilang mga indibidwal na kabahagi ng higit na masaklaw na realidad at komunidad. Hindi inihihiwalay kung gayon ng mga fans ang kanilang identidad bilang mga mag-aaral, maging mga propesyonal, sa pagyakap ng identidad bilang mga fangirl. Bagaman may ilang gawaing ipinagpapaliban sa puntong magbukas ang oportunidad na makita ang idolo-tulad ng pagsasantabi ni Jane sa nakaiskedyul na recitation at long test sa Trigonometry upang makita ang idolong madalang magawi sa lugar (Jadaone, 2020)—-mas madalas na inuuna ng mga tagahanga ang realidad bilang mag-aaral na bukod sa makapagpamalas ng katalinuhang laging inaalis mula sa kanila, ay ginagawa ring inspirasyong makapagbubukas ng mas malaking pagkakataong masuportahan ang idolo (Lar. 15).

Salungat sa madalas na pagkilala sa kanila sa midya bilang indibidwal na madalang magtagumpay sa kani-kanilang mga karera, (Stanfill, 2011, para. 3.5), marubdob na pinatutunayan ng fandom na sa espasyong ito nakalagak ang ilan sa pinakamatatagumpay na propesyonal na mula sa iba't ibang larang. Mula sa katotohanang inihayag ni Coppa sa kaniyang pananaliksik ukol sa mga fan ng Sci-Fi partikular na ng Star Trek na marami sa mga tagahanga ang sumuong sa pagiging mga scientist, computer programmer, at mathematician (2008, para. 2.3), nasa balikat pa rin ng mga kontemporanyong tagahanga online na patunayan ang mga personal na tagumpay na labas sa mundo ng paghanga (Lar. 16). 
Omg 16 eps nga lang. Ending na pala talaga $\stackrel{2}{6}$ 龺 next week after exam ko ilalaban ko na yan.

Wait lang punta muna ako sa klase HAHAHAHHAHAHA

balik ako >s

Translate Tweet

12:59 PM - Jan 15, 2021 - Twitter for Android

Larawan 15. Bukod sa direktang paglalatag ng responsabilidad bilang mag-aaral sa pamamagitan ng mga tweet na ibinabahagi (gee, 2021; KAYE, 2021), wikang kinakasangkapan din ng espasyo ang 'IA' o inactive (lin | ia na naman jusko; $r($ ia) \#; maybe studying; rin | ia) na siyang babala sa kapuwa fan ng pansamantalang pagtalikod sa espasyo upang tugunan ang ilang personal na gawain.

\section{1. . $^{28}$ rpwk}

Hi. I'm Liz. I'm 22 years old and I recently graduated from college with my undergraduate degree in writing. I work two jobs and I love 1D.

Quote with who and what you are. I'm trying to prove a point.

9:24 AM - Dec 9, 2018 - Twitter for iPhone

107 Retweets $\mathbf{2 9 2}$ Quote Tweets $\mathbf{8 4 1}$ Likes

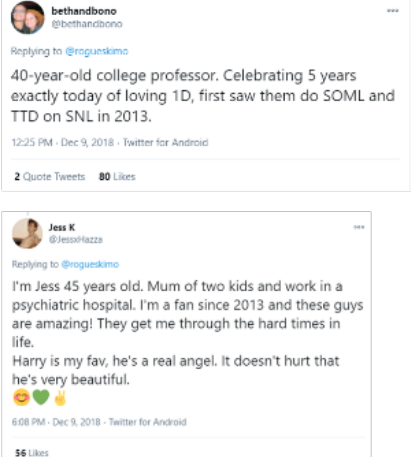

Larawan 16. Mga pagkakataong isinasabalikat ng mga fan ang responsabilidad na isiwalat sa madla ang pagiging matagumpay na miyembro ng mas malawak na lipunan (L, 2018; bethandbono, 2018; Jess K, 2018).

Hindi naman natutuldukan ang paggigiit ng intelek ng mga tagahanga sa paglalantad lamang ng kani-kanilang personal na tagumpay. Maihahanay rin sa kanilang nag-uumapaw na kakayahang kumilala sa higit na masaklaw na realidad sa labas ng mundo ng paghanga ang boluntaryong pakikianib sa mas malawak na kilusang panlipunan at sistematikong mobilisasyong naglalayon ng mabungang kawanggawa (Lar. 17) (Jenkins, 2018, p.51). Sa pagyakap ng mga naturang gawain nakapag-aangkin ang fandom ng kolektibong responsabilidad na epektibong naipapahiwatig ang kanilang hangaring tumindig bilang komunidad na hindi lamang lubog sa pantasya kundi komunidad na kayang makapagbunsod ng gawaing maipagmamalaki (Cook \& Hynes, 2014, p. 90). 

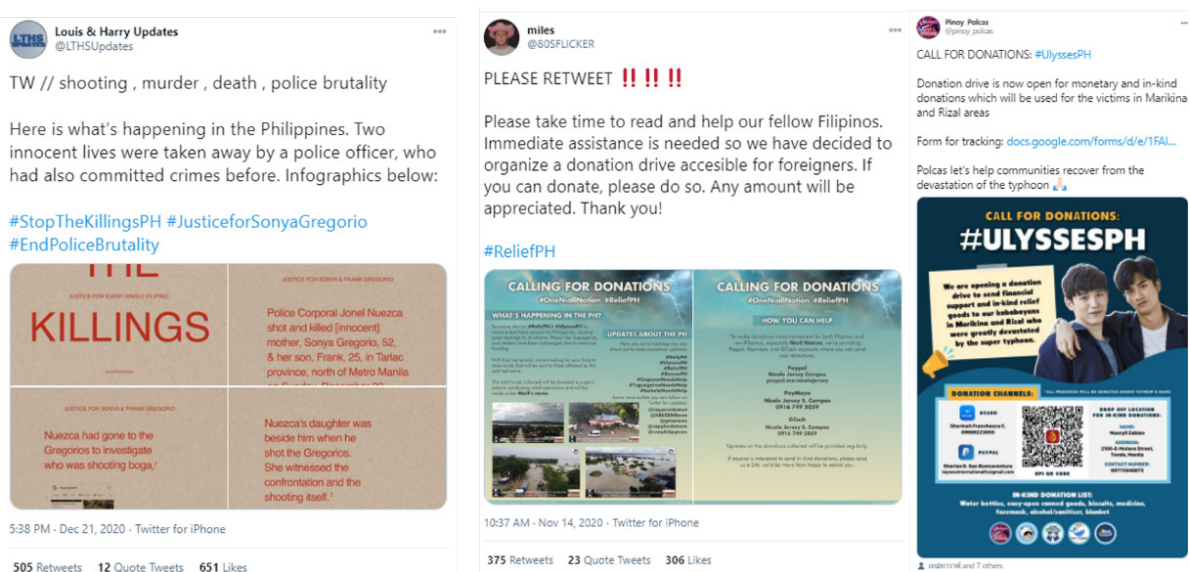

Larawan 17. Ilang halimbawa ng pakikisangkot na naisasagawa ng mga fangirl na mababakasán ng kolektibo at sistematikong pagtugon sa suliraning kinahaharap ng higit na mas malawak na komunidad na kinabibilangan (Louis \& Harry Updates, 2020; miles, 2020; Pinoy_Polcas, 2020).

\section{Ang Kasalimuotan ng Pagiging Fangirl}

Sa birtuwal na espasyong kinalalagakan ng mga tagahanga, palasak ang makakita ng pagpapahayag na sumusunod sa anyong "I hate men, but I love [idol]" (Lar. 18). Bukod sa kinikilala ng kataga ang umiiral na ugnayan sa pagitan ng babaeng tagahanga at madalas lalaking idolo, sabay nitong napanghahawakan ang makapangyarihang paggigiit ng isang babae na yakapin ang paggana ng kaniyang "affect" sa pagpapasiyang kumiling sa isang obheto ng paghanga nang hindi isinasantabi ang pagmamalay sa higit na malawak na dinamikang pangkasarian na kinahinaharap sa loob ng patriyarkal na lipunan. Sa pagsailalim sa ganoong pagtitimbang, na tila nasa anyong panggigisa pa nga ng sarili dahil sa umiiral na kontradiksiyon, masalimuot na napagtatagpo ng mga fangirl ang prosesong intelektuwal at ang pagganang emosyonal na hindi nagsasantabi ng kanilang hangarin at pagnanasa. Sa pagkilala sa tagibang na relasyon at sa sabay na paggalugad nito, naiaalok ng fandom mismo ang alternatibong pagkilala sa kanilang komunidad bilang repleksibo na umuugat sa kakayahang kilalanin at siyasatin ang mga sarili.

Hindi tuloy madaling ikahon sa isang pagkakakinlanlan at representasyon lamang ang fandom na madalas naisasagawa ng midya na tila ba naninindigan sa unibersal na pagkakaintindi sa isang "fan" (Scott, 2019, p. 227). Dahil bahagi ng praktika ang self-awareness at self-critique na sabay na nakapagbubunsod ng maramihan, sala-salabid, at sapinsaping pagbabago, masalimuot ang artikulasyong naisasagawa ng mga fangirl hinggil sa kanilang identidad na maipagpapalagay na hindi kayang saklawin ng representasyong nakasanayan na ng midya. Hamon sa gayong 


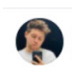

fra is a sunflower

do you ever go..

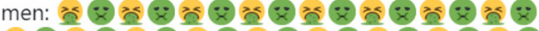

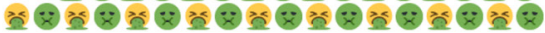

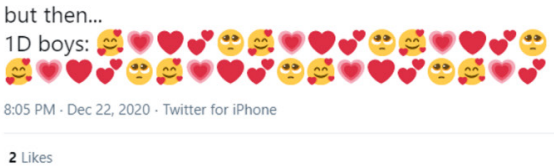

R\&l content

girls on twitter about men: i hate them so much $\because \ddot{q}$

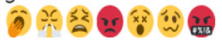

girls on twitter about louis tomlinson and harry styles:

$\because 00 \% 0$ 충

3:34 AM · Sep 1,2020 - Twitter for Android

148 Retweets 6 Quote Tweets 488 Likes

Larawan 18. Palasak na pahayag hinggil sa masalimuot na dinamika ng kasarian na masasaksihan sa paghangang nakalagak sa espasyong online (fra is a sunflower, 2020; h\&l content, 2020).

patingkarin sa pamamagitan ng iba't iba ring pamamaraan ang kanilang panloob na dinamikang makapaghahawan ng landas ng higit pang pagunawa sa kanilang komunidad. Sa pagtalikod sa binary (Scott, 2019, p. 30)-o paghahain lamang ng tunggaliang emosyonal laban intelektuwal, mature at childish, hegemoniko at subersibo, at pagkilala sa fluidity ng fandom na nagbabagong-hubog sa bawat sandaling nailulunsad ng anuman, makapagtataguyod ng alternatibong pagkilala sa masalimuot na mundo ng kababaihang paghanga. Sa pamamagitan ng pagiging malay sa alok na "counternarratives," "multiplicities," at "competing visions" ng fandom mismo (Scott, 2019, p. 50), mapapalawak hindi lamang ang kategorisasyon ng pagiging fangirl, kundi pati na rin ang nosyon ng pagkababaeng palaging ikinakabit dito. 


\section{MGA SANGGUNIAN}

[@golden0ius]. (2018 April 17). honestly imagine being THAT pretentious just because you have the privilege to stalk Harry everywhere and go to every one [Tweet]. Twitter. https://twitter.com/ goldenOuis/status/985958021854650368

: - 8 [@nttsthetic]. (2021 January 13). this is not cool. Apparently there are two fans who have been waiting for tay in front of his house [Tweet]. Twitter. https://twitter.com/nttsthetic/ status/1349341160968310785

HLina ${ }^{28} \bigcirc$ 's Nobody is listening [@91CHERRYOTB]. (2021 January 14). Here's a thread of larries you should follow [Tweet]. Twitter. https://twitter.com/91CHERRYOTB/status/1349443674774642691

@finefilm. (2021a January 12). GoodFellas (1990 dir. Martin Scorsese If you were told you were only allowed to watch one movie for the rest [Tweet with photo attached]. Twitter. https://twitter.com/finefilm/ status/1348987264160948227

@finefilm.(2021bJanuary 12). Harrystyleswatchlist—athreadwith ten of hisfavouritefilms \& hiscommentary [Tweet with photo attached]. Twitter. https://twitter.com/finefilm/status/1348987231852179456

\#WeBlockAsOne:KathNiel fans lead campaign vs. trolls. (2020, May 16). Squeeze. https://squeeze.ph/ weblockasone-kathniel-fans-lead-campaign-vs-troll

1D Facts [@1DirectionFQS]. (n.d.) Tweets [Twitter profile]. Retrieved January 14, 2021 from https://twitter. com/1DirectionFQS

ABS-CBN News. (2014, May 24). Bandila: One Direction fans cry as tickets get sold out [Video]. Youtube. https://www.youtube.com/watch?v=9x7R6un12AU

ABS-CBN News. (2015, March 23). Local celebs enjoy One Direction concert [Video]. Youtube. https://www. youtube.com/watch?v=aG3AgEsiqoc.

ALY BIRTH!!!!!! [@jisaucekrist]. 2021 January 3. Here's my entry \#SingtoSoSexy [Tweet with photo attached]. Twitter. https://twitter.com/jisaucekrist/status/1345745355980193792

ara (@singtosubs). (2020a September 22).8. Tuesdays with Morrie by Mitch Albom. he uploaded an ig story about him reading this book $n$ he was seen [Tweet with photo attached]. Twitter. https://twitter.com/ singtosubs/status/1308411541134376966

ara (@singtosubs). (2020b September 22). just singto prachaya ang his love for books! [aka singto's books list] - a thread [Tweet with photo attached]. Twitter. https://twitter.com/singtosubs/ status/1308395817150029825

arche [@apollohalo]. (2020 December 26). Not all larries are straight/cis/white stop calling us that [Tweet]. Twitter. https://twitter.com/apollohalo/status/1342750019519041536

ash | y\&B zayn's gf [@curlycuntx]. (2020 November 17). SPOLIER: ONE DIRECTION ARE IN MAJOR TALKS OF GOING ON AN 18 MONTH HIATUS WHICH WILL INVITABLE EXTEND TO 5 [Tweet]. Twitter. https:// twitter.com/curlycuntx/status/1328547808362647552

Bacon-Smith, C. (1992). Enterprising women: Television fandom and the creation of popular myth. University of Pennsylvania Press.

Baker, L. I. (2017). The surface of women. Transformative Works and Cultures, 24. http://dx.doi.org/10.3983/ twc.2017.879 
Barrios, J. (2015). Kung bakit lagi kong pinapanood ang mga pelikula ni Sharon Cuneta. In R. B. Tolentino \& G.C. Devilles (Eds.), Kritikal na espasyo ng kulturang popular (pp. 279- 285). Ateneo de Manila University Press.

bethanbono [@bethandbono]. (2018 December 9. 40-year-oold college professor. Celebrating 5 years exactly today of loving 1d, first saw them do SOML and TTD on SNL [Tweet]. Twitter. https://twitter. com/bethandbono/status/1071621946465546241

Blake, Y. (2020, March 10). For the love of fangirls [Video]. Youtube. https://www.youtube.com/ watch?v=0B543Zkqq88

Bourdieu, P. (1984). Distinction: A social critique of the judgment of taste. Routledge.

Braid, B. (2014). Fassination, fandom, and the crisis of hegemony: Michael Fassbender's Performance of masculinity and the female gaze. In A. Chauvel, N. Lamerichs \& J. Seymor (Eds.) Fan studies: Researching popular audiences (pp. 73-84). Inter-Disciplinary Press.

celly ${ }^{29}$ [@91LOUH4BIT]. (2021 January 14). i'm being ignored here bye [Tweet]. Twitter. https://twitter. com/91LOUH4BIT/status/1349502295965253632

celly²9 [@91LOUH4BIT]. (2021 January 14). ikaw grr chz miss youuu [Tweet]. Twitter. https://twitter. com/91LOUH4BIT/status/1349503151456485377

Cook, A., \& Hynes, D. (2014). From the football terraces to the television screen: Gender and sexuality and the challenges of online fan communities. In A. Chauvel, N. Lamerichs \& J. Seymor (Eds.) Fan studies: Researching popular audiences (pp. 85-96). Inter-Disciplinary Press.

Coppa, F. (2008). Women, Star Trek, and the early development of fannish vidding. Transformative Works and Cultures, 1. https://doi.org/10.3983/twc.2008.044

Coscarelli, J. (2020 December 25). How pop music fandom became sports, politics, religion, and all-out war. The New York Times. https://www.nytimes.com/2020/12/25/arts/music/pop-music-superfansstans.html?auth=link-dismiss-google1 tap\&smid=tw-share

de Certeau, M. (1984). “General introduction," In The Practice of Everyday Life (S. Rendall, Trans). University of California Press.

Denden || ia [@singkittles]. (2021 January 8). good morning ice hahahaha ganito kapag stress sa acads [Tweet]. Twitter. https://twitter.com/singkittles/status/1347374689191043073

Denden || ia [@singkittles]. (2021 January 8). gusto ko mag apply bilang p.a. ni singto-personal asawa [Tweet]. Twitter. https://twitter.com/singkittles/status/1347373231276056577

dree [@kitsingverse). (2021 January 7). PATULUGIN NIYO NA NGA TO [Tweet]. Twitter. https://twitter.com/ kitsingverse/status/1347183232052125709

fi [@perachayas]. (2020 June 29). 1. sotus or sotus s? [Tweet]. Twitter. https://twitter.com/perachayas/ status/1277526274894749696

fi [@perachayas]. (2020 June 29). 2. first thoughts/impression on arthit's hazing team? [Tweet]. Twitter. https://twitter.com/perachayas/status/1277526278006927361

fi [@perachayas]. (2020 June 29). Random questions about \#SotusTheSeries and \#SotusSthe Series; a thread [Tweet]. Twitter. https://twitter.com/perachayas/status/1277526270570455040

Flegel, M., \& Roth, J. (2014). Mommy porn and regurgitated fiction: The silencing of women in fan debates about pulled to publish fan fiction. In A. Chauvel, N. Lamerichs \& J. Seymor (Eds.) Fan studies: Researching popular audiences (pp. 55-71). Inter-Disciplinary Press. 
fra | wants a rn. [@hazzaonpoutine]. (2020 December 22]. Do you ever go... men: but then... 1D boys: [Tweet]. Twitter. https://twitter.com/hazzaonpoutine/status/1341354124500217857

Gee [@kitsingtowx]. (2021 January 15). Omg 16 eps nga lang.Ending na pala talaga next week after exam ko ilalaban ko na yan [Tweet]. Twitter. https://twitter.com/kitsingtowx/status/1349912736675766272

GMA News. (2014, December 20). 24 oras: Kauna-unahang One Direction world store sa Pilipinas, dingsa ng fans [Video]. Youtube. https://www.youtube.com/watch?v=L2NsuCbt1GU

GMA News. (2015, March 21). Mahigit 30,000 fans ng One Direction, inaasahang daragsa sa concert ngayong gabi [Video]. Youtube. https://www.youtube.com/watch?v=rPDukLETaJ8

H\&L content [@larrysuits]. (2020 September 1). girls on twitter about men: $i$ hate them so much girls on twitter about louis tomlinson and harry styles [Tweet]. Twitter. https://twitter.com/larrysuits/ status $/ 1300517430905778176$

haileylovesIhh [@INFINITYLOU].(2021 January12).okaysoifiwearpearlsim dating harrystyles?Gotit[Tweet with photo attached]. Twitter. https://twitter.com/INFINITYLOU/status/1349007378319106052

Hjarpe, K.M. (2008). Rebyu ng akdang Cyberspaces of their own: Female fandoms online by R. Burry. Transformative Works and Cultures, 1. https://doi.org/10.3983/twc.2008.036

ice <3 | ks au [@ice_kswhipped]. (2021 January 8).aga aga nito denden ah good morning [Tweet]. Twitter. https://twitter.com/ice_kswhipped/status/1347374146645225474

I Love Arguing with Antis [@_Josiann] (2020 December 27). I still don't know what cis means, can you explain [Tweet]. Twitter. https://twitter.com/_Josiann/status/1342981917289967617

ilyKS [@kitsingmyluv].(2021 January4).this is 100\% me25y/oand singlesincebirthEven tholknowkpop since 10 years ago and [Tweet]. Twitter. https://twitter.com/kitsingmyluv/status/1345955134321246209

incorrect Larry [@incorrectLarry). (2020 December 11). Harry: hey, we can't go in there, it says 18+ and there's only 2 of us. Louis: you're so fucking [Tweet]. Twitter. https://twitter.com/incorrectLarry/ status/1337223887260700673

J. [@singtocentavo]. (2021 January 8).BHAHAHAHAHAHAHAHAHAHA SELOS KA SIGE PAKASAL TAYO BUKAS, SAN MO GUSTO? EUROPE? HAHAHAHA ILYSM [Tweet]. Twitter. https://twitter.com/singtocentavo/ status/1347425011456385024

Jadaone, A. (Director). (2020). Fan girl [Film]. Black Sheep Productions.

Jaz is listening [@confettintheam] (2021 January 12). Zayn Malik, nagsagawa ng birthday party sa Jolibee at McDonald's! [Tweet with photo attached]. Twitter. https://twitter.com/confettintheam/ status/1348941638291189761

Jenkins, H. (1992). Textual poachers: Television fans and participatory culture. Routledge.

Jenkins, H. (2018). Fandom, negotiation, and participatory culture. In P. booth (Ed.) A companion to media fandom and fan studies (pp. 37-55). Wiley Blackwell.

Jess K [@JessxHazza]. (2018 December 9). I'm Jess 45 years old. Mum of two kids and work in a psychiatric hospital. I'm a fan since 2013 [Tweet]. Twitter. https://twitter.com/JessxHazza/ status/1071708058747846657

Julia [@hrawberry]. (2018. May 16). Just bc ur rich parents can pay foryou to have barricade at every show and literally stalk harry [Tweet]. Twitter.https://twitter.com/hrawberry/status/996702157112934401

Kaye [@BabyPERAYA0419]. (2021 January 15).Wait lang punta muna ako sa klase HAHAHAHAHHAHAHAHA balik ako [Tweet]. Twitter. https://twitter.com/BabyPERAYA0419/status/1349944345886027777 
Kompare, D. (2018). Fan curators and the gateways into fandom. In M.A. Click, \& S. Scott_(Eds.) The Routledge companion to media fandom (pp. 107-113). Routledge.

L [@rogueeskimo]. (2018 December 9). Hi. I'm Liz. I'm 22 years old and I recently graduated from college with my undergraduate degree in writing [Tweet]. Twitter. https://twitter.com/rogueskimo/ status/1071576282582974464

Larsen, K., \& Zubernis, L. (2013). Introduction. K. Larsen, \& L. Zubernis (Eds), Fan culture: Theory and practice (pp. 1-13). Cambridge Scholars Publishing.

Leah D's rach [@KILLMYSWEAT]. (2021 January 7). there's no better way to promote a movie than making director and cast date, except this whole thing is giving [Tweet]. Twitter. https://twitter.com/ KILLMYSWEAT/status/1346884239934058498

Lexi 『s y\&b [@hairyween]. (2021 January 13). If you actually stan harry you wouldn't invade his privacy/ stalk him [Tweet]. Twitter. https://twitter.com/hairyween/status/1349050019681361920

lin | ia na naman jusko [@offgunsbb]. (n.d.) Tweets [Twitter profile]. Retrieved January 20, 2021 from https://twitter.com/offgunsbb

lynn [@saltedpeaches]. (2020 December 5). AU in which KongArt are cockroaches who survived the apocalypse. Cockroaches are the only species left alive, but even though [Tweet]. Twitter. https:// twitter.com/saltedpeaches/status/1335228474496192512

Lorenz, T., Browning, K., \& Frenkel, S. (2020, November 6). Tiktok teens and K-pop stans say they sank Trump rally. The New York Times. https://www.nytimes.com/2020/06/21/style/tiktok-trump-rallytulsa.html

Louis \& Harry Updates [@LTHSUpdates]. (2020 December 21). TW// shooting, murder, death, police brutality Here is what's happening in the Philippines. Two innocent lives were taken away by [Tweet with photo attached]. Twitter. https://twitter.com/LTHSUpdates/status/1340954759981719552

mai [@F00L4LOUIS]. (2021 January 14). i miss u sooo much more [Tweet]. Twitter. https://twitter.com/ F00L4LOUIS/status/1349503319346089985

mai [@F00L4LOUIS]. (2021 January 14). sino nagiingnore sa asawa ko [Tweet]. Twitter. https://twitter.com/ F00L4LOUIS/status/1349503003527507968

maya [@wmsugars]. (2021 January 7). harry is a fan of the artist JEAN-MICHEL BASQUIAT, mentioning him in the 2020 beauty papers video "what's my name?" [Tweet with photo attached]. Twitter. https:// twitter.com/wmsugars/status/1347153826827042816

maya [@wmsugars]. (2021 January 7). the artistic interests \& collection of harry styles - a thread [Tweet with photo attached]. Twitter. https://twitter.com/wmsugars/status/1347153684086419459

Meggers, H. (2013). Discovering the authentic sexual self. K. Larsen, \& L. Zubernis (Eds), Fan culture: Theory and practice (pp. 57-80). Cambridge Scholars Publishing.

miles [@80SFLICKER]. (2020 Novermber 14).PLEASE RETWEET!!! Please take time to read and help our fellow Filipinos. Immediate assistance is needed so we have decided [Tweet with photo attached]. Twitter. https://twitter.com/80SFLICKER/status/1327440585247002624

MIST// rest. [@skperawhipped]. (2021 January 2). jollibee or mcdo [Tweet]. Twitter. https://twitter.com/ skperawhipped/status/1345344357692440576

MIST// rest. [@skperawhipped]. (2021 January 2). kfc or wendy's [Tweet]. Twitter. https://twitter.com/ skperawhipped/status/1345344391708246017 
MIST// rest. [@skperawhipped]. (2021 January 2). since my tl is full of food, im gonna make a this or that, food edition. U can qrt [Twet]. Twitter. https://twitter.com/skperawhipped/status/1345344127370625025

Mizoguchi, A. (2008). Reading and Living yaoi: Male-Male Fantasy Narratives as Women's Sexual Subculture in Japan [Doctoral dissertation, University of Rochester]. University of Rochester Libraries.

Muntean, N., \& Petersen, A.H. (2009). Celebrity Twitter: Strategies of intrusion and disclosure in the age of technoculture. M/C Journal, 12 (5). https://doi.org/10.5204/mcj.194

NO CONTROL [@RevilSakura]. (2015. April 27). "you only like 1d cause of their looks" COUGHT YEP SO DAMN HOT AHAHAHAHA COUGH \#BestFandomCA2015 Directioners" [Tweet with photo attached]. Twitter. https://twitter.com/RevilSakura/status/592483813726425088

Penley, C. (1992). Feminism, psychoanalysis, and the study of popular culture. In L. Grossberg, G. Nelson \& P. Treichler (Eds.), Cultural studies now and in the future (pp. 479-500). Routledge.

Pinoy_Polcas [@pinoy_polcas]. (2020 November 14). CALL FOR DONATIONS: \#UlyssesPH Donation drive is now open for monetary and in-kind donations which will be used for [Tweet with photo attached]. Twitter. https://twitter.com/pinoy_polcas/status/1327432930914754560

Rappler [@rapplerdotcom. (2021 January 13). LOOK: as of 9:30 am on Wednesday, January 13, \#CayetanoStopUsingBTS is one of Twitter Philippines' trending topics, following reports of [Tweet]. Twitter. https://twitter.com/rapplerdotcom/status/1349170691401261066

r(ia);i maybe studying [@perxaya]. (2021 January 11). She's dating the gangster but it's KS [Tweet with photo attached]. Twitter. https://twitter.com/perxaya/status/1348652590263767041

r(ia);i maybe studying [@perxaya]. Tweets [Twitter profile]. Retrieved January 20, 2021 from https:// twitter.com/perxaya

ria \'s coacoac [@H4BIT_MEDIC1NE]. (2020 December 27). hi! Cis means you still go with the gender that you were assigned from birth! [Tweet]. Twitter. https://twitter.com/H4BIT_MEDIC1NE/ status/1342982699712131072

Rin | ia [@singtuanbby]. Tweets [Twitter profile]. Retrieved January 20, 2021 from https://twitter.com/ singtuanbby

ryn ara [@Kristalloidal]. (2021 January 8). Nagtatampo pa rin ako sa'yo kasi nagpakasal ka sa iba pero sige na nga okay na, hmp, thank you and ily [Tweet]. Twitter. https://twitter.com/Kristalloidal/ status/1347424760121094146

sarah is listening [@babielouis28]. (2020 August 4). All fans of harry don't often agree about anything but we do when it's about how ugly his merch is [Tweet]. Twitter. https://twitter.com/babielouis28/ status/1290322631028178947

Scott, S. (2019). Fake geek girls: Fandom, gender, and the convergence culture industry. New York University Press.

Sedgwick, E. K. (1994). Tendencies. Routledge.

shifa loves nina [@UNFXCKVIBEZ]. (2021 January 7). Media: harry styles out with his new love interest olivia wilde me, an intellectual, knowing that it's just a stunt [Tweet with a photo attached]. Twitter. https:// twitter.com/UNFXCKVIBEZ/status/1346896200084058112

Stanfill, M. (2011). Doing fandom, (mis) doing whiteness: Heteronormativity, racialization, and the discursive construction of fandom. Transformative Works and Cultures, 8, https://doi.org/10.3983/ twc.2011.0256 
sunflower [@sunflow72295713]. (2021 January 19). @Harry_Styles I really fucking love you but like. Wear. A. mask. You have a fan base and a lot of [Tweet]. Twitter. https://twitter.com/sunflow72295713/ status/1351479234942799872

sunflower [@sunflow72295713]. (2021 January 19). I'm saying this because it was irresponsible of you to not wear a mask. I'm really disappointed [Tweet]. Twitter. https://twitter.com/sunflow72295713/ status/1351479238474387456

Swati $\mathrm{H}^{\mathrm{H} 2}$ is missing Louis [@otbhxrry]. (2020 December 8). A thread of masterposts for all new Larries/ Twarries/Antis [Tweet]. Twitter. https://twitter.com/otbhxrry/status/1336258455057788928

teddy. [@ENBYLWT]. (2021 January 13). -louis tomlinson's journal. 2010-2020<3 [Tweet with photo attached]. Twitter. https://twitter.com/ENBYLWT/status/1349367719754403840

The fan meta reader. (2015). Fandom in/as contact zone, by tea-and-liminality [Blog Post]. https://wp.me/ p4YnOf-90

ThisDayin1DHistory [@todays1dhistory]. (n.d.) Tweets [Twitter profile]. Retrieved January 14, 2021 from https://twitter.com/todays1dhistory

Trinidad, A.I. (2018). Fandom, fangirling, at stan culture: Pagkilala sa kasalukuyang kultura ng kabataang paghanga at paghahangad sa loob ng bansa. Katipunan: Journal ng mga Pag-aaral sa Wika, Panitikan, Sining at Kulturang Filipino, 3 (1), 148-188. https://journals.ateneo.edu/ojs/index.php/ katipunan/article/view/2853

Trinidad, A.l. (2021). Hinggil sa realidad ng pagiging fangirl. Young critics circle film desk. https:// yccfilmdesk.wordpress.com/2021/01/06/hinggil-sa-realidad-ng-pagiging-fangirl/

TWC Editor. (2010). Exploring the body. Transformative Works and Cultures, 5. https://doi.org/10.3983/ twc.2010.0244

yel [@singtosiasm). (2021 January 8). @singkittles @kitsingverse @singtocentavo good morning naman sa tatlong to na bungad at laman ng aking tl keep clowning mga bbs [Tweet]. Twitter. https://twitter. com/singtosiasm/status/1347323230508392448

Zsila, A., \& Demetorvics, Z. (2017). The boys' love phenomenon: A literature review. Journal of Popular Romance Studies, 6, 1-16. https://www.jprstudies.org/2017/04/the-boys-love-phenomenon-aliterature-reviewby-agnes-zsila-and-zsolt-demetrovics/ 


\section{Notes}

${ }^{1}$ Umiiral ang ganitong nosyon hindi lamang sa pag-iisip ng karaniwang madla kundi pati na rin sa mga unang iskolar na nakapaglunsad ng Fan Studies bilang akademikong larang. Sa pagsasakasaysayan ng disiplinang naglalayong umunawa sa praktis at gawi ng mga tagahanga, matagal nang establisado ang fandom bilang isang komunidad na dominanteng binubuo ng mga kababaihan. Sa kasalukuyan, kung kailan umiigting ang pag-aaral sa mga komunidad na nakalagak sa espasyo ng internet (Larsen \& Zubernis, 2013, p. 4), umiiral pa rin ang paniniwalang sentral ang mga babae sa pag-unawa ng fandom.

${ }^{2}$ Tumutukoy sa likhang-sining ng mga tagahanga na isinasailalim sa paiba-ibang kasarian ang mga karakter na hinalaw mula sa obheto ng fandom. Ilan sa mga kategoryang nakapaloob dito ang mpreg na nagiging posible sa piksiyonal na mundo ang pagdadalang-tao ng isang lalaki at ang femslash na tinatransporma ang mga orihinal na lalaking karakter bilang mga babae.

${ }^{3}$ Malawak na nasaksihan ang patunay rito sa pag-trend $\mathrm{ng}$ \#CayetanoStopUsingBTS kamakailan nang ianunsiyo ni Cayetano ang pagpapangalan sa mayoryang di-umano ay ineetsapuwera sa kongreso (Rappler [@rapplerdotcom], 2021). 


\section{Grant Support Details}

Author Contributions: All research activities and writing were done by A. A. I Trinidad. The author has read and agreed to the published version of the manuscript.

Funding: The author received no specific funding for this work.

Acknowledgements: Pinasasalamatan ng may-akda ang lahat ng kababaihang tagahanga, lalo na ng bandang One Direction, ng ship na Larry Stylinson at ng Thai BL Imaginary Couple na Krist-Singto, na malayang nagpapahayag ng kanilang pagmamahal sa iniidolo nang hindi isinasantabi sa praktika ng fangirling ang pagpapagana ng kani-kanilang intelek at ahensiya.

Conflict of Interest: The author declares no conflict of interest.

\section{About the Author}

Si ANDREA ANNE I.TRINIDAD ay nakapagtapos ng kursong AB-MA Literature (Filipino) sa Pamantasang Ateneo de Manila at kasalukuyang instruktor sa Kagawaran ng Filipino, Paaralang Humanidades sa parehong unibersidad. Nakatuon ang kaniyang pag-aaral sa kulturang popular partikular na sa personal na interes sa larang ng Fan Studies at Popular Romance. (Email address: atrinidad@ateneo.edu) 